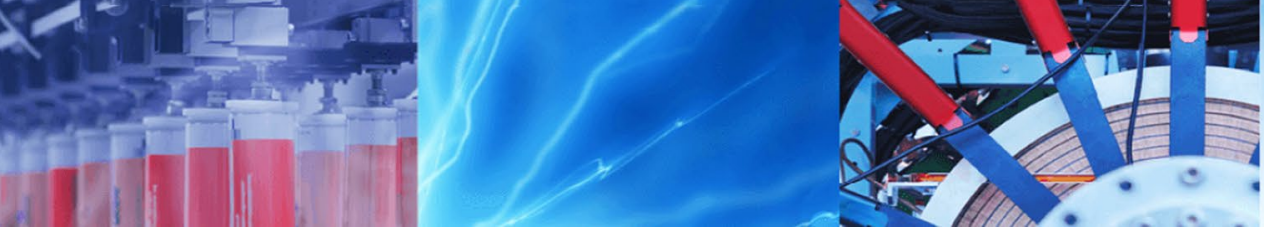

Research Article

\title{
Pruned Kd-tree: a memory-efficient algorithm for multi-field packet classification
}

\author{
M. Rafiee ${ }^{1}(\mathbb{D}) \cdot$ M. Abbasi $^{1}(\mathbb{0}$
}

Received: 3 September 2019 / Accepted: 29 October 2019 / Published online: 4 November 2019

(c) Springer Nature Switzerland AG 2019

\begin{abstract}
Packet classification is a basic process in most network-based packet processing systems. The key operation in this process is to match the packet header against the rules defined in a rule-set and, finally, to find the best matching rule. One of the well-known algorithms for packet classification is the Kd-tree algorithm. This algorithm produces a binary tree using the tuples created by the length of the prefix of the source and destination IP addresses of the rules. The tree is intended to classify the packets. The efficiency of the mechanism for producing and searching the binary tree in this algorithm is affected by two major disadvantages, namely, redundant nodes and redundant accesses during the search. The former increases memory consumption and the latter slows down packet classification. The proposed idea in this article is to prune redundant nodes by sorting the rules corresponding to each tuple in the tree nodes. The experimental results suggest that the throughput rate of the pruned $\mathrm{Kd}$-tree is $43.64 \%$ higher than the $\mathrm{Kd}$-tree. Also, by pruning the structure of the $\mathrm{Kd}$-tree, our proposed method could at best reduce $24 \%$ of the memory consumed for the storage of the data structure of the Kd-tree.
\end{abstract}

Keywords Packet classification $\cdot$ Acceleration $\cdot$ IP networks $\cdot$ Kd-tree $\cdot$ Pruning $\cdot$ Memory

\section{Introduction}

The process of dividing network packets into different flows in Internet routers and firewalls is called packet classification. Packet classification specifies to which flow each packet belongs. Classification is based on matching one or more fields from the packet header against the corresponding values from the rules of the classifier. The most important fields of packet headers that are used for packet classification include source IP address, destination IP address, protocol type, source port number, and destination port number. When a packet is received, the table of rules is searched for a matching rule in accordance with the fields of the packet header. In addition to the valid values for the header fields, each rule specifies the action to be applied to the packet [1, 2]. The action determined by the matching rule will be applied to the packet. Since a packet may be matched by several rules, the action determined by the rule with the highest priority will be performed. For example, one priority can be the length of the prefix of the source or destination IP addresses or the order of the appearance of a rule in a rule set [3-5].

One of the well-known packet classification algorithms is the Kd-tree algorithm [6]. This algorithm has a balanced binary tree structure. The tree represents the rules. Each node in this tree, therefore, contains rules that are partly similar to each other. The rules that are mapped onto a node in the tree have identical tuples. That is, the prefixes of the source and destination IP address fields of the rules mapped onto a tree node will have the same length. It should be noted that the Kd-tree algorithm is a

M. Abbasi, abbasi@basu.ac.ir; M. Rafiee, m.rafiee@alumni.basu.ac.ir| ${ }^{1}$ Department of Computer Engineering, Engineering Faculty, Bu-Ali Sina University, Hamedan, Iran. 
combination of tree-based and tuple space methods. In other words, a binary search is performed on the tuple space.

This study proposes an optimized version of the Kd-tree packet classification algorithm by pruning the redundant nodes of the tree. The most important criteria for the efficiency of the algorithm are packet classification time and the space required to store the structure of the $\mathrm{Kd}$-tree algorithm. For this purpose, we first examine the tree structure of the Kd-tree algorithm as well as how this algorithm classifies packets. Then, we will show how pruning the tree structure created by the rules and changing the packet classification model in the pruned tree will reduce the time for traversal as well as packet classification. Also, we closely examine how to reduce the space needed to store the tree structure of the Kd-tree algorithm.

The structure of the article is organized as following. First, we shall review related works on the structure of the $\mathrm{Kd}$-tree algorithm as used in tuple space search as well as the use of markers in this algorithm. In the third section, the proposed method for pruning the Kd-tree and producing a pruned $\mathrm{Kd}$-tree is examined. Implementation and evaluation of the proposed method are explained in Sect. 4. The final section of the paper is dedicated to concluding and presenting solutions for further development of research in this field.

\section{Related works}

\subsection{Decision tree based packet classification algorithms}

Decision-tree based algorithms $[5,7,8]$ are the most popular algorithms for packet classification. Their success is owned by the key idea of recursively cutting the search space into smaller sub-spaces, each of which corresponding to a child of node in a decision-tree. Such a recursive terminates when the number of rules in tree nodes becomes lower than a predefined threshold.

To classify an incoming packet, information of certain fields of its header are extracted and then used to traverse the tree. During traversal from root to leaf nodes, the algorithm stores the best matching rule based on its specific policy.

There are a few variations of the decision tree that differ on the method of constructing the tree via cutting the search space and the way of traversing the tree for classifying packets. For example, HiCuts [7] constructes the tree via multiple evenly-spaced cuttings on a single dimension at each iteration. HyperCuts [8] differs from HiCuts only in allowing multiple dimensions to be cut concurrently to moderate the height of the constructed tree. To resolve the memory blow up problem caused by rule replication of HyperCuts, HyperSplit [5] uses non-equal cuts for controlling the memory usage. As shown in Fig. 1a, HiCuts algorithm cuts the search space into two equal-sized subspaces. The Rule 2 and Rule 3 are replicated in the respective subspaces. By aligning the cuttings at the edges of the rules, the HyperSplit algorithm is able to reduce rule replication (Fig. 1b). However, HyperSplit still cannot eliminate all rule replication, especially for complex rule sets.

However, this algorithm is not as fast as HyperCuts. A key solution to this problem is to implement the algorithm on FPGA [9]. In this hardware implementation of HyperSplit, a pipelined architecture accelerates the classification process. However, due the limited resources of the programmable devices limits the extensibility and customizability of the hardware packet classifiers. Also, the considerable design costs of hardware classifiers makes their performance to cost ratio smaller than that of the corresponding software classifiers. For this reasons, software packet classifiers are more interested.

The common issue in using software packet classifiers is their inability in achieving maximum speed and minimum memory usage, simultaneously. Therefore, the memory usage of fast tree-based packet classification algorithms may grow exponentially as the number of rules increases. In this paper we show how using simple but effective tricks in pruning redundant tree nodes would reduce the memory consumption of the algorithm and increase the classification speed.

\subsection{Kd-tree algorithm based on tuple space search}

In spite of the large number of rules in a classifier, often the majority of prefixes have the same length. Using this fact, the rules of a classifier are divided into separate groups based on their prefix length.

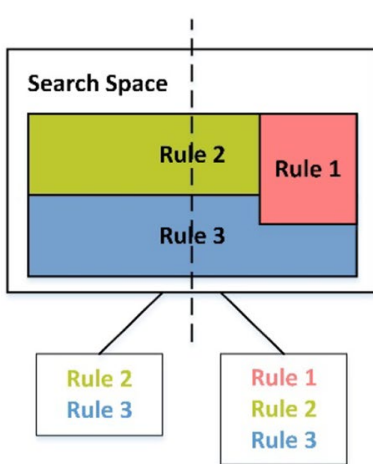

(a)

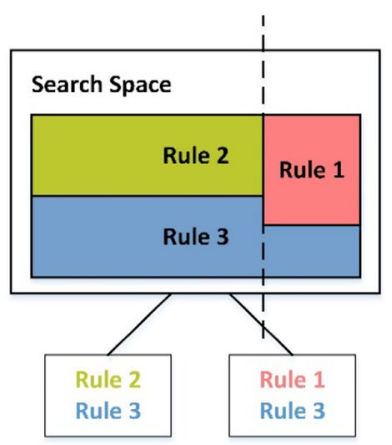

(b)
Fig. 1 a Unaligned cut point (causing replication of Rule 2); b Aligned cut point to the boundary of Rule 1 and Rule 2 (reducing replication) 
It is clear that the number of rules that are placed in a category with the same prefix length is less than the total number of rules. For example, consider a classifier in a traditional router that sends packets based on the destination IP address. Such a classifier, regardless of the number of rules, can have up to 32 distinct groups. In a two-dimensional classifier in which rules are defined based on the source and destination IP addresses, a maximum of 1024 separate groups can be conceived. However, many of the tuples may have no specific rule. In general, if you consider a $d$-dimensional classifier, each rule is mapped onto a vector with a set of $d$ integers in which the ith integer denotes the length of the prefix of the ith field of the rule. A vector with $d$ integers is called a tuple. A set of tuples created by the classifier is called tuple space. The modest algorithm for tuple space search is to check all the tuples.

\subsection{Definitions}

A tuple $T$ is a vector of $d$ integers that is defined as $T . v e c[1], T . v e c[2], \ldots, T . v e c[d]$. In this definition, T.vec[i] denotes the integer value corresponding to the ith item in $T$. An $f$ rule matches a tuple if and only if $\forall i, 1 \leq i \leq d$ and the length of the ith field of $f$ is exactly $T \cdot v e c[i]$.

The space of tuple $T$ can be divided into three partitions, i.e., LongerTuple, ShorterTuple, and IncomparableTuple. Suppose two tuples $T$ and $T_{a}$ and let $T_{a} \neq T$ $\left(\exists i, 1 \leq i \leq d, T_{a} \cdot v e c[i] \neq T . v e c[i]\right) . T_{a}$ is a tuple in the LongerTuple partition of $T$ if $\forall i, 1 \leq i \leq d, T_{a} . v e c[i] \geq T \cdot v e c[i]$. Also, if $\forall i, 1 \leq i \leq d, T_{a} \cdot \operatorname{vec}[i] \leq T \cdot \operatorname{vec}[i]$, then $T_{a}$ is a tuple in the ShorterTuple partition of $T$. Otherwise, $T_{a}$ is a tuple in the IncomparableTuple partition of $T$.

To perform a binary search in the tuple space, a binary tree should be produced using tuples. For this purpose, each tuple is mapped onto a unique value called SuperKey which specifies the position of the tuple on the tree. The SuperKey of tuple $T$ is generated by joining all the elements in T.vec in a rotational order. For this purpose, a discriminator is used to determine the place where the elements should be joined. $S K_{T . d i s}$ represents the SuperKey of $T$ and dis index represents the discriminator. Therefore, $S K_{T . d i s}=T . v e c[\operatorname{dis}] T . v e c[$ dis +1$], \ldots, T . v e c[d] T . v e c[1] T . v e c$ $[2], \ldots, T . v e c[$ dis -1$]$. For example, $S K_{(3,0) .1}=30$ and $S K_{(3,0) .2}=03$. Since there are $d$ elements in T.vec, tuple $T$ can have $d$ SuperKeys that may begin at any element $1,2,3, \ldots, d$.

As SuperKeys are integers, they can be easily sorted. As a result, the tuples can be sorted by their SuperKeys. Assuming dis as the discriminator, $T_{a}$ is smaller than $T$ if $S K_{T_{a} \text {.dis }}<S K_{T \text {.dis }}$. Otherwise, $T_{a}$ would be greater than $T$. For example, let dis $=1$. Then the tuple $(3,0)$ is greater than the tuple $(2,2)$ because $S K_{(3,0) .1}>S K_{(2,2) .1}(30>22)$. If the discriminator of all the tuples equals 2 , the tuple
$(3,0)$ would be smaller than the tuple $(2,2)$ because of $S K_{(3,0) .2}<S K_{(2,2) .2}(03<22)$.

\subsection{The structure of the Kd-tree algorithm}

Rules that are mapped onto a tuple can be stored in a hash table. Tuples can be considered as points in a multidimensional space. Over the past three decades, many data structures have been developed for organizing multidimensional objects, including Kd-tree [10], KDB-tree [11], R-tree [12], R+-tree [13], and R* -tree [14]. Among these data structures, $\mathrm{Kd}$-tree provides a simple and convenient method. More information on this structure can be found in several recent studies [15-18].

In our proposed method, each node of a Kd-tree holds a tuple $T$, and two pointers are connected to the right and left sub-trees. According to the explanations given in Sect. 2.3, all the tuples of the left-hand sub-tree of a tuple $T$ are smaller than $\mathrm{T}$ itself whereas all the tuples in the righthand sub-tree of T are greater than T. Note that the tuples of the left-hand sub-tree of $T$ belong to the ShorterTuple or IncomparableTuple of $T$. Also, the right-hand tuples of $T$ belong to the LongerTuple or IncomparableTuple of $T$.

To simplify the formation of a Kd-tree algorithm, assume that only the fields of source and destination IP address of the rules are checked. The sample classifier works with the ten rules in Table 1. In this table, the first column shows the number of rules. The second and third columns represent the source and destination IP addresses, respectively. The fourth column represents the tuple corresponding to the rule. For example, in the case of Ro rule, the number of the bits of the source IP address prefix is 4 and that of the destination IP address prefix is 3 . Therefore, the tuple corresponding to this rule is $(4,3)$.

Table 1 An example of the rules of a classifier

\begin{tabular}{llll}
\hline Rule & Source IP address & $\begin{array}{l}\text { Destination IP } \\
\text { address }\end{array}$ & Tuple \\
\hline R0 & $1010^{*}$ & $011^{*}$ & $(4,3)$ \\
R1 & $1010^{*}$ & $0000^{*}$ & $(4,4)$ \\
R2 & $100^{*}$ & $101^{*}$ & $(3,3)$ \\
R3 & $1^{*}$ & $010^{*}$ & $(1,3)$ \\
R4 & $10110^{*}$ & $1000^{*}$ & $(5,4)$ \\
R5 & $11^{*}$ & $011^{*}$ & $(2,3)$ \\
R6 & $10111^{*}$ & $01001^{*}$ & $(5,5)$ \\
R7 & $*$ & $0 *$ & $(0,1)$ \\
R8 & $101^{*}$ & $01^{*}$ & $(3,2)$ \\
R9 & $11^{*}$ & $1111^{*}$ & $(2,4)$ \\
\hline
\end{tabular}

*Symbol in all patterns of source IP address and destination IP address denotes a part of the address which is unimportant to the classifier engine 
To select the root node or a sub-tree of the Kd-tree, SuperKeys are created to sort the tuples. The tuples are first sorted according to their SuperKeys, and then the middle tuple in the sorted list is selected as the root node or a sub-tree of the Kd-tree. Tuples with SuperKeys smaller than that of the selected tuple are assigned to the lefthand sub-tree of the tuple, and the rest of the tuples are assigned to the right-hand sub-tree of the root tuple. This process is repeated for the tuples assigned to the left-hand and right-hand sub-trees. In other words, to select the root of the left-hand and right-hand tree, the existing tuples are once again sorted using a new discriminator, and the middle tuple of the list is selected as the root of the sub-tree. The following formula is used to determine the discriminator field in creating the SuperKey of tuples at the $L$ th level:

dis $=(L \bmod d)+1$

In the above equation, $d$ denotes the number of dimensions of the classifier. In fact, with the help of Eq. (1), fields $1,2,3, \ldots, d$ are selected in rotation as the discriminator. The tree shown in Fig. 2 is based on the source and destination IP addresses in Table 1. In this tree, the elliptical nodes correspond to the tuples in the fourth column of Table 1. Also, the rules belonging to each tuple are shown with the symbol R[]. For example, tuple $(3,2)$ has an R8 rule.

In this example, the tuples are initially sorted by the discriminator dis $=1$. Accordingly, the SuperKeys corresponding to the tuples in Table 1 are obtained and sorted in the form of $(0,1),(1,3),(2,3),(2,4),(3,2),(3,3),(4,3),(4,4),(5$, $4),(5,5)$. The middle tuple $(3,2)$ is selected as the root node of the Kd-tree. The tuples $(0,1),(1,3),(2,3),(2,4)$ fall under the left-hand sub-tree of the root and the tuples $(3,3),(4$, $3),(4,4),(5,4),(5,5)$ under the right-hand sub-tree. Then the discriminator dis $=2$ is selected, and the tuples in the left-hand and right-hand sub-trees are sorted again. The sorted sequences are $(0,1),(1,3),(2,3),(2,4)$ and $(3,3),(4$, $3),(4,4),(5,4),(5,5)$. The middle tuples $(1,3)$ and $(4,4)$ are selected as the root nodes of the left-hand and right-hand sub-trees, respectively. By repeating this process, the tree of the $\mathrm{Kd}$-tree algorithm is constructed.

\subsection{Search in Kd-tree algorithm and the misjudged problem}

This section examines an example of searching in the $\mathrm{Kd}$ tree. Assume a packet with the source IP address (1001 0011101011111111111100110100 ) and the destination IP address (0010 011100001011001101011111 1111). Given the rules in Table 1 and the corresponding Kd-tree depicted in Fig. 2, the act of classification for this packet will be as following. In the search process, BstMatch variable is used. This variable holds the first matching rule until the search is finished and a leaf node is reached.

First, traversal starts from the root node of the Kd-tree, which is the tuple $(3,2)$ in this example. Therefore, this tuple should be checked. To check $(3,2)$, if it contains a rule according to the number of the prefix bits of the source and destination IP addresses, it will be matched against the input packet. Therefore, the packet matches the rules in the form of $\left(100^{*}, 00^{*}\right)$. In this example, the input packet does not conform to any rule because the prefixes of $R 8$ cannot be matched with the prefixes of the packet. Since this tuple has returned the result of mismatch, the tree is further traversed on its left-hand child. In the next step which consists of matching against the tuple $(1,3)$, the search algorithm uses 1 bit of the prefix of the source IP address and 3 bits of the prefix of the destination IP address for examining the rules in the tuple $(1,3)$. At this stage, the algorithm cannot find any matching rule. Due to the lack of matching rules, therefore, traversal is led to the left-hand sub-tree of this tuple. Then the rules in the last tuple, i.e. $(0,1)$, are checked. In this step, Rule 7 is returned as the best matching rule because the algorithm has already reached a leaf node.

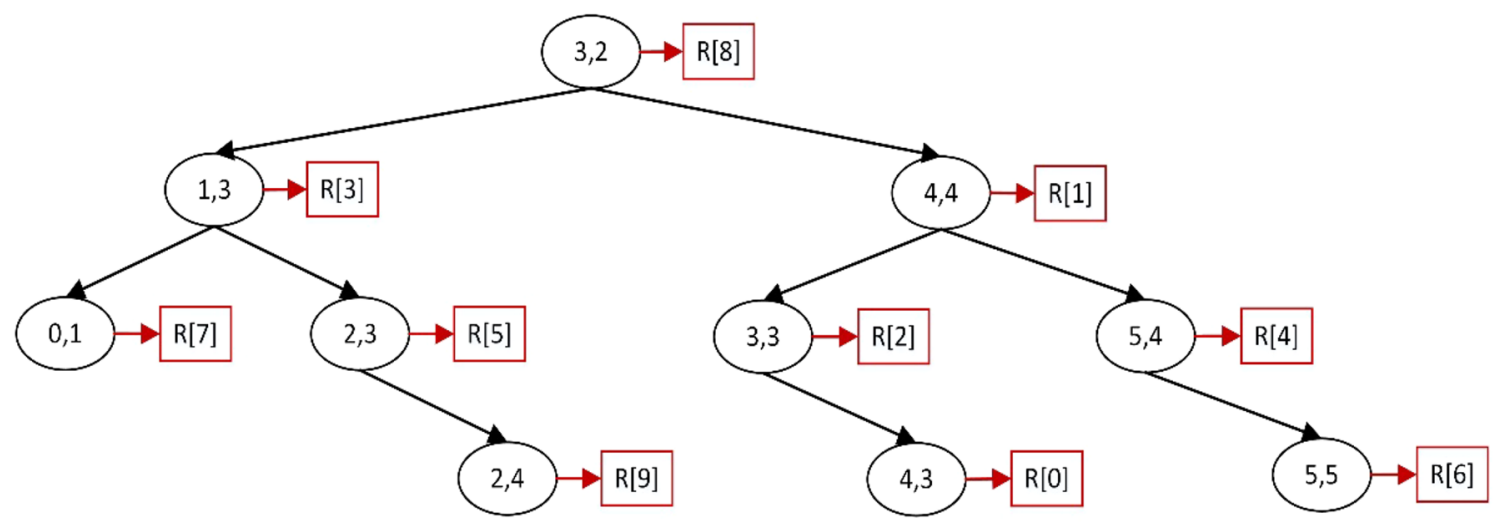

Fig. 2 The basic Kd-tree produced from the rules in Table 1 
As the above description shows, performing a traversal for packet classification by the Kd-tree is not flawless. For example, suppose an input packet for the matching operation has a source IP address of (1100 0011101100100111 $011011111111)$ and a destination IP address of (1111 1011010011101111111101000000 ). At the root of the tree, the packet has not been matched by any of the rules of $(3,2)$ and, as a result, traversal continues at the left-hand sub-tree. In the next step, the input packet does not match any of the rules in the tuple $(1,3)$ and again traversal continues at the left-hand sub-tree of the tuple $(1,3)$. The root tuple of this sub-tree is $(0,1)$ whose rules do not match the packet. Finally, the search is over without any result. In a linear search, however, the packet would easily match the rule R9. A solution to this problem are markers. The next section explains how a marker is used in the basic algorithm.

\subsection{Use of markers in the Kd-tree algorithm}

When a search is not successful on a tuple, the search algorithm continues the traversal on the left-hand subtree and removes all the tuples of the right-hand subtree from the traversal. To avoid this problem, which is called the misjudged problem, every tuple should retain information about the rules of its right-hand sub-tree. For example, the tuple $(1,3)$ must have a marker that produces $\mathrm{R} 5$ rule in the tuple $(2,3)$. Also, the tuple $(1,3)$ must have a marker of $\mathrm{R} 9$ rule related to the tuple $(2,4)$. Figure 3 shows the $\mathrm{Kd}$-tree of the rules in Table 1 along with added markers. Markers in the tuples are indicated by $M[\ldots]$.

In the search for the best matching rule from the previous example, traversal cannot find any matches in the tuple $(3,2)$ and continues on the left. When matched against $(1,3)$, the search is successful due to the presence of the R9 marker. Finally, the search algorithm continues to traverse the right-hand sub-tree and reports R9 rule in the tuple $(2,4)$ as the best matching rule.

\section{Pruned Kd-tree algorithm}

In this section, we describe the pruned Kd-tree method which significantly improves the Kd-tree algorithm. Assume an input packet with fields that can be best matched by R6 in Fig. 3 (a source IP address of (1011 $1111001001010001111110011101)$ and a destination IP address of (01001101 1010000101101111 1011 1111)). Figure 4 shows the tree traversal path to find the best matching rule. The tree nodes to be examined are shown in shaded shapes. Traversal starts from the root node. To search for the best matching rule for this packet by means of the Kd-tree algorithm, in each node a certain length of the source and destination IP address of the packet is compared with the corresponding prefixes from the rules mapped onto the tuple of the node. For example, in the root node which contains the tuple $(3,2)$, the input packet is compared with only 3 bits of the prefix of the source IP address and 2 bits of the prefix of the destination IP address of the existing rules. Therefore, as the figure shows, all the rules in a tuple must be checked. In this example, traversal starts from the root towards the right-hand child of $(4,4)$. Then the tuple $(5,4)$ and, finally, the tuple $(5,5)$ are traversed. Finally, as the traversal path reaches a leaf node, the rules of that node are also examined and $\mathrm{R} 6$ rule is reported as the result. In this example, 14 nodes are checked in the traversal path. In the following, we will show that the idea of pruning a Kd-tree can significantly reduce the number of accessed nodes. Thus, the search speed in this algorithm increases significantly.

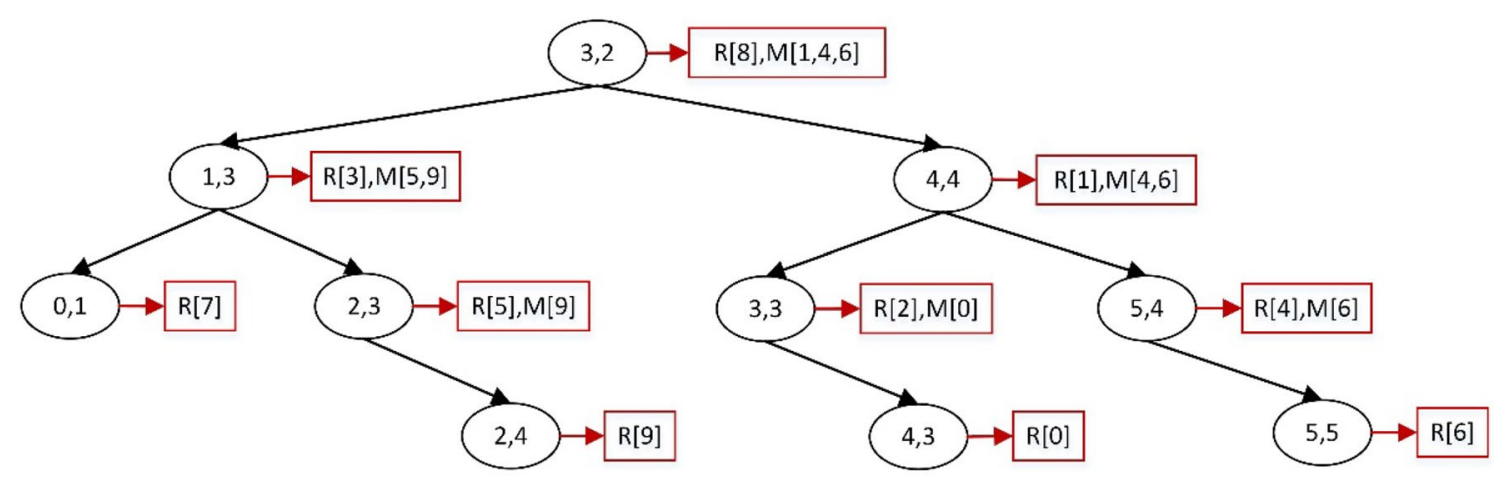

Fig. 3 The Kd-tree with markers of the rules from Table 1 


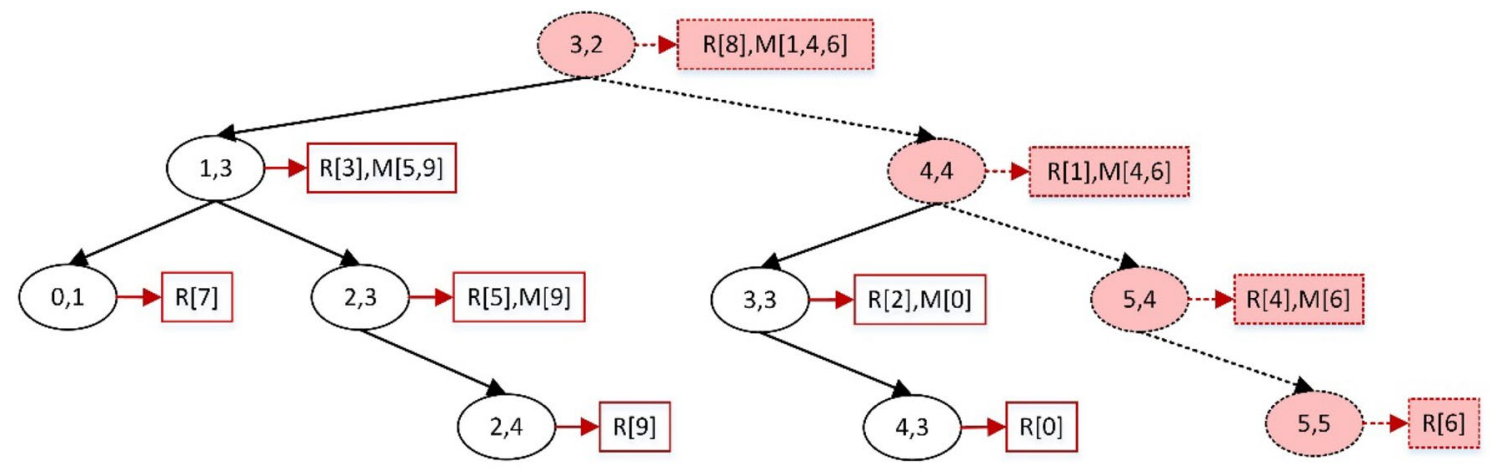

Fig. 4 Traversal of the Kd-tree

\subsection{Suggested techniques for pruning a Kd-tree}

Our examination of Kd-trees created by various rules resulted in the following ideas for tree pruning.

Technique 1 Pruning the right-hand leaves

In the Kd-tree data structure, by replacing the markers of a node with the rules that correspond to the tuple of the right child of the node and matching the entire prefix bits against the input packet, we can prune the right child if it is a leaf node.

In other words, all the rules in the right child node will also exist in the parent as markers. If the right child node is a leaf node, it can be pruned by transferring the rules to the parent; therefore, instead of matching the marker, it is necessary to match the rules against the input packet.

Obviously, pruning some of the tree's redundant nodes will reduce the memory needed to store the tree structure. On the other hand, sometimes the tree's depth will decrease with the removal of leaf nodes. Another advantage of pruning a tree is to reduce the number of memory accesses during the traversal.

Technique 2 Sorting by the priority of rules

If in each node corresponding to a tuple all the existing rules have been sorted in descending order according to the prefix length, memory access will be significantly reduced when searching for the best matching rule. The rules are sorted based on the length of the sum of the source and destination IP address prefixes.

Indeed, sorting the rules by prefix length makes it possible that, when the input packet is matched with the first rule during the traversal, the search could be finished at that tuple and continue on the right-hand sub-tree of the node, if any, to find matching rules corresponding to longer tuples. Because this method does not need to necessarily check all the nodes in the tuple, the number of memory accesses and the time for packet classification is reduced.

\subsection{The Structure of pruned Kd-tree algorithm}

In this section, using the techniques mentioned above, we prune a Kd-tree. Algorithm 1 describes how to create a pruned Kd-tree. The input and output of this algorithm are $\mathrm{Kd}$-tree and pruned $\mathrm{Kd}$-tree. To prune the tree, all nodes are checked (lines 1 through 7). If the examined node is a leaf node, it will be pruned as long as it is the right child of its parent (lines 2 to 6 ).

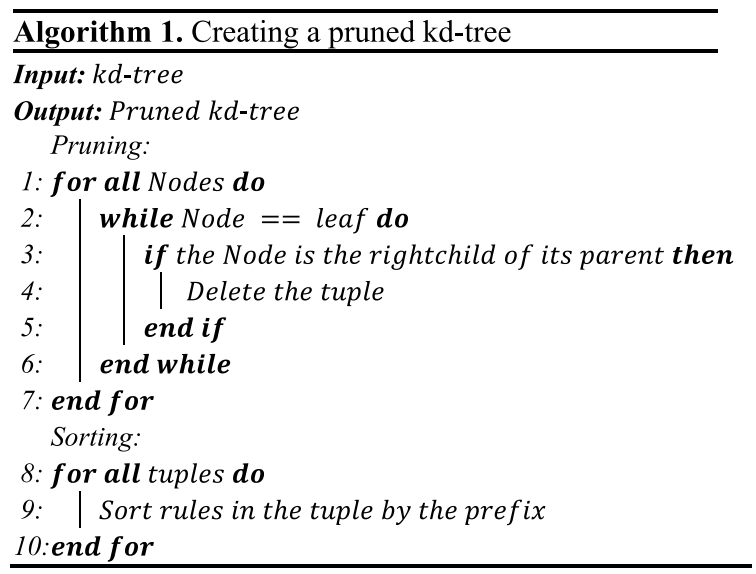

After the tree is pruned, it is necessary to sort the rules in the nodes corresponding to each tuple. For this purpose, all the nodes of the tuples are examined, and their rules are sorted by the prefix length in descending order (lines 8 to 10).

Figure 5 represents the $\mathrm{Kd}$-tree corresponding to Table 1. In this tree, the leaf nodes that are the right child of their parent are marked in gray. In the next step, these 


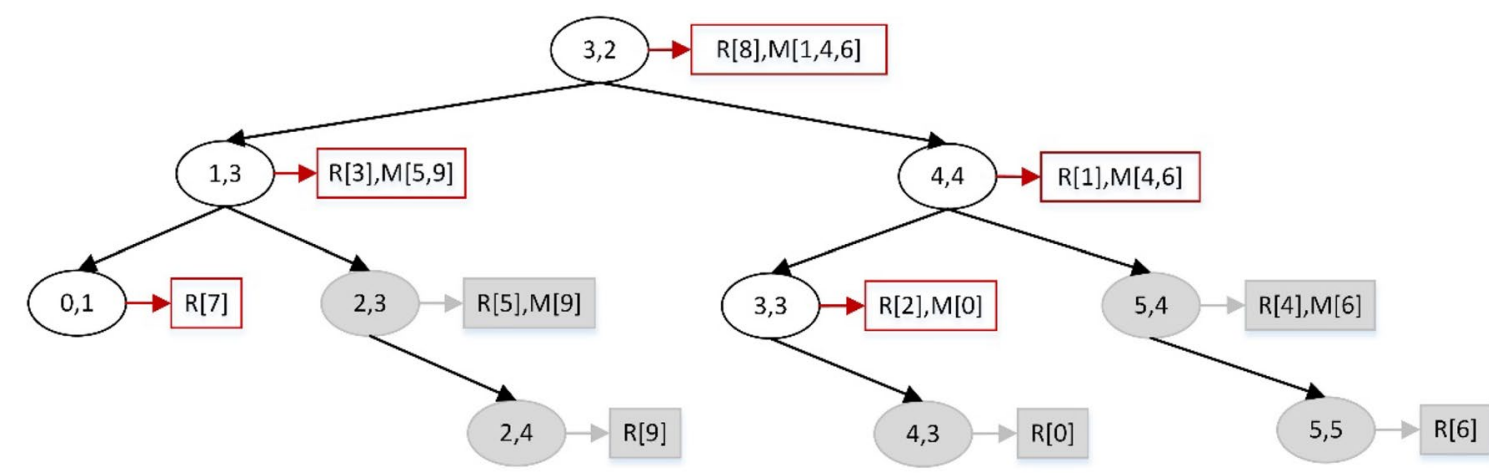

Fig. 5 Nodes to be pruned by Kd-tree algorithm

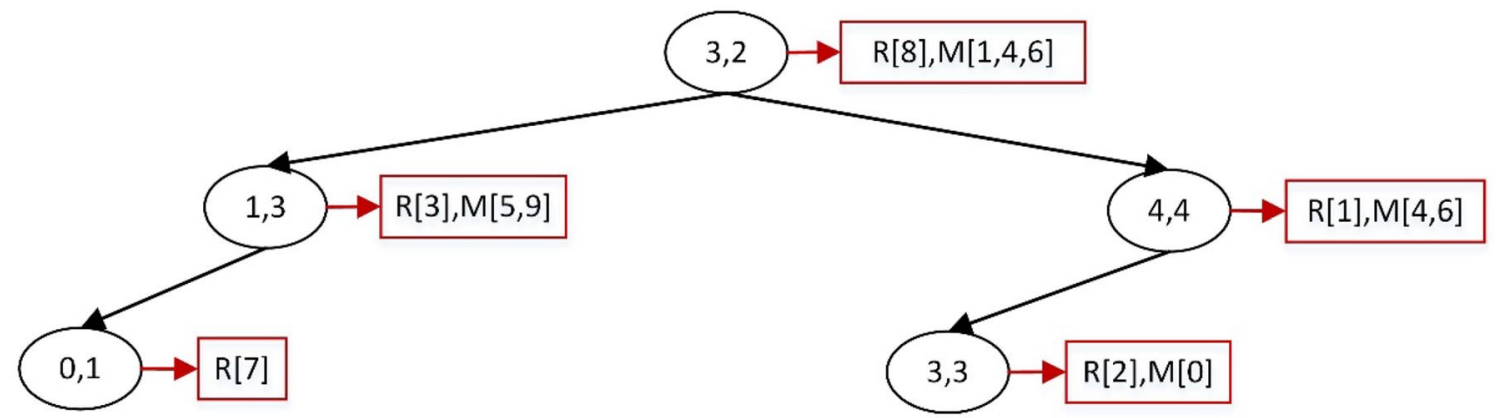

Fig. 6 The Kd-tree after pruning some nodes

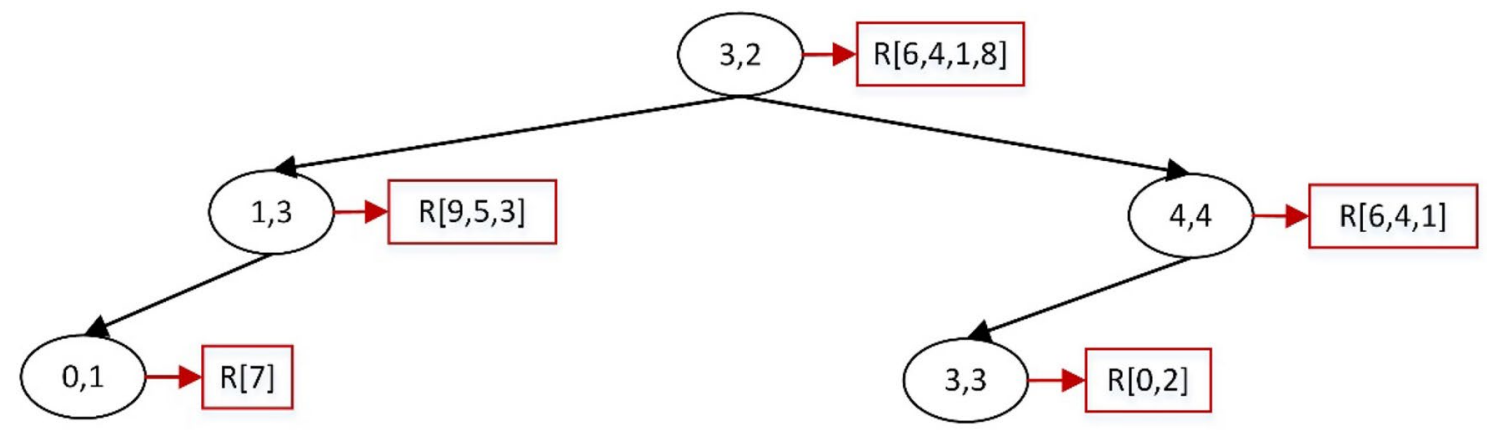

Fig. 7 The tree produced by the implementation of the proposed method of pruned Kd-tree on the rules in Table 1

nodes are eliminated, resulting in the removal of 12 nodes from the tree. Figure 6 shows the structure of the Kd-tree after pruning.

Figure 7 shows the final version of the tree shown in Fig. 6. In this trie, all of the markers in each node are added to the rules set R. Next, these rules are sorted descending by their prefix length. For example, the root node which has the markers $1,4,6$ and the rule $R 8$, is converted to $R[1,4,6,8]$. In this node, rule $R 6$ has the longest prefix as compared to others. R4 is the second of the longestprefix rules. The rules are sorted in this way in all of the tree nodes. 


\subsection{Search in a pruned Kd-tree}

Search in a pruned Kd-tree is done as follows. Traversal starts from the root and the rules in each tuple node are checked linearly. The rules are examined in their entirety, i.e. the full length of the prefix of source and destination IP addresses, source and destination port numbers, and protocol. If the packet matches a search rule, the search in the rules of that tuple is stopped and directed to the right-hand sub-tree of the tuple.

Algorithm 2 shows how to search and classify packets based on the data structure of the pruned tree. The input of this algorithm is the rule set $R$, the pruned $\mathrm{Kd}$-tree $T$, the tuples, and the header of input packets. The output is rulesIndexArray which holds the index of the best matching rule of every incoming packet. Packet classification starts from the root node (line 2). Initially, BMR variable that holds the best matching rule for a packet is set to Null (line 3). The traversal of the tree continues until a leaf node is reached (lines 4 through 13). On the traversal path, the different fields of the rules in the tuples are compared with the input packet. If the packet matches a rule, the number of that rule will be stored in rldx (line 6). Then, if the packet matches a rule in the tuple, the result will be stored in $B M R$ and traversal will be directed towards the right child; otherwise, the left child will be traversed (lines 7 to 12). At the end of the traversal, the result of packet classification is stored in rules/ndexArray which is an array used for holding the results (line 14).

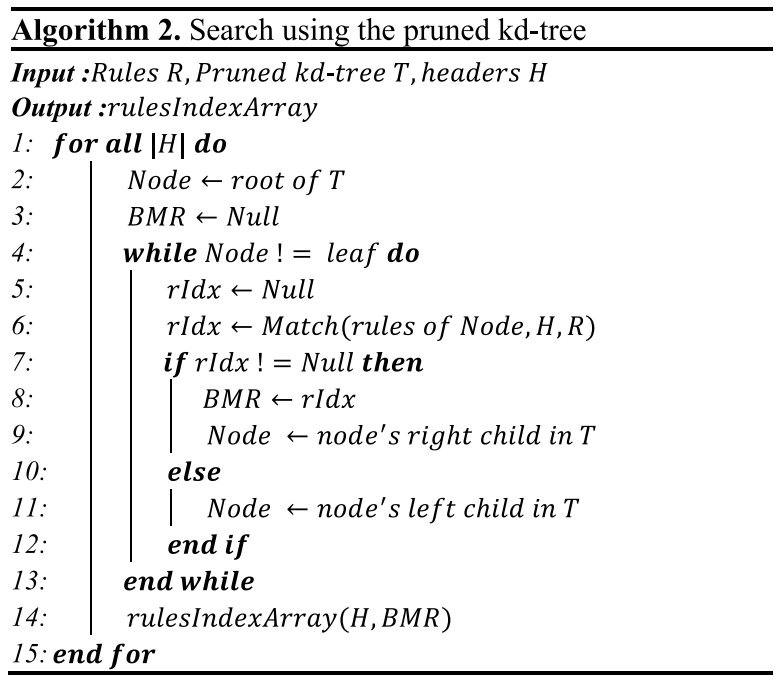

Table 2 compares the number of memory accesses required for classifying same packets using basic Kd-tree and its enhanced version.

Figure 8 illustrates searching this prund Kd-tree for first packet of Table 2. It is assumed that the packet matches R6. Multiple nodes and rules that are examined on the traversal path are shown in red. This search, which would otherwise need 14 memory accesses in the Kd-tree algorithm, is performed with only four memory accesses in the proposed method.

Table 2 Number of memory-accesses required for Kd-tree and pruned Kd-tree

\begin{tabular}{|c|c|c|c|c|}
\hline \multicolumn{2}{|l|}{ Input packet } & \multicolumn{2}{|c|}{ Algorithm } & \multirow[t]{2}{*}{ BMR } \\
\hline Source IP address & Destination IP address & Kd-tree & Pruned Kd-tree & \\
\hline 10111111001001010001111110011101 & 01001101101000010110111110111111 & 14 & 4 & R6 \\
\hline 10110010000110011001100000000000 & 10000000000000110101001100111001 & 9 & 6 & R4 \\
\hline 11000011101100100111011011111111 & 11111011010011101111111101000000 & 14 & 7 & R9 \\
\hline
\end{tabular}

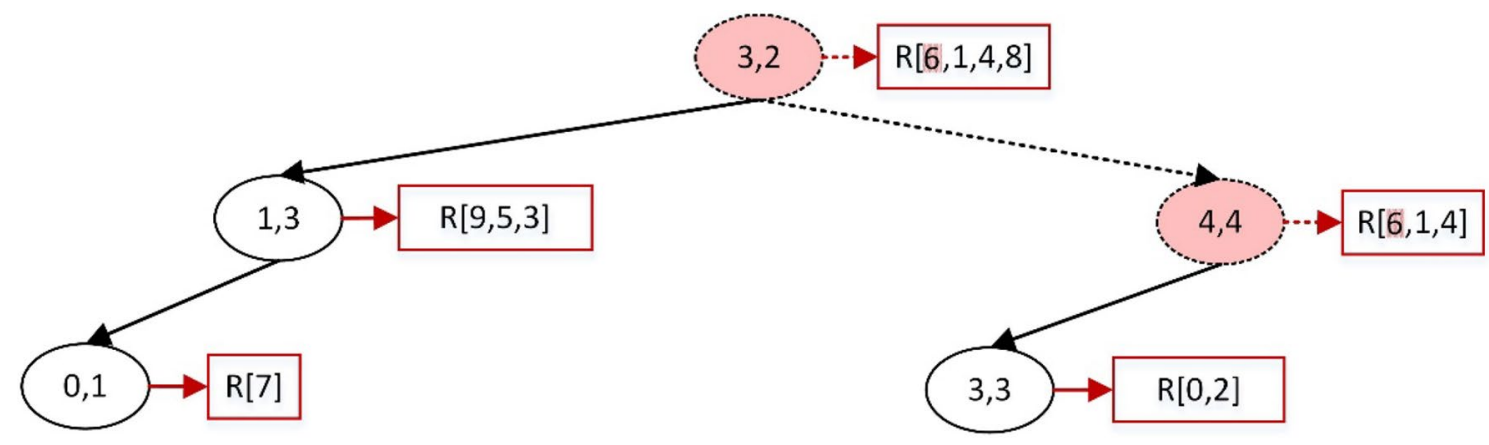

Fig. 8 Search using the pruned Kd-tree algorithm 
Table 3 System specifications

\begin{tabular}{ll}
\hline Specification & Processor \\
\hline Name & Intel Core i7-740QM \\
Clock speed & $1733 \mathrm{MHz}$ \\
L1 data cache & $4 \times 32 \mathrm{~KB}$ \\
L1 inst. cache & $4 \times 32 \mathrm{~KB}$ \\
L2 cache & $4 \times 256 \mathrm{~KB}$ \\
L3 cache & $6 \mathrm{MB}$ \\
Main memory & 4 GB DDR3 \\
Operation system & Windows 7 ultimate, 64-bit \\
\hline
\end{tabular}

In classifying the second packet of Table 2, the Kdtree algorithm examines rule 8 in the tuple $(3,2)$ and for its inconsistency checks the marker of this tuple. Hence, the packet is examined against the markers of rule 1 and rule 4 , and due to matching with the marker of rule 4 , the subsequent search is directed to the right subtree. Then, the packet is compared with rule 1 and marker 4 in the tuple $(4,4)$, which finally is matched with rule 4 . The number of memory accesses in classifying this packet using Kd-tree is nine. But, in pruned Kd-tree, the packet is first examined against rules 4 and 6 in the tuple (3, 2 ), and then is compared with rules 4 and 6 , with only six memory accesses. Similarly, the number of required memory accesses for classifying the third packet of Table 2 is computed and presented. In these examples, the number of memory accesses of the proposed algorithm is lower than that of the basic algorithm.

Table 4 The number of memory accesses with the ACL rule set

\begin{tabular}{|c|c|c|c|c|c|c|c|c|c|}
\hline \multirow[t]{2}{*}{ Packet } & \multirow[t]{2}{*}{ Access memory } & \multicolumn{4}{|c|}{ Kd-tree algorithm } & \multicolumn{4}{|c|}{ Pruned Kd-tree algorithm } \\
\hline & & $1 \mathrm{k}$ & $5 \mathrm{k}$ & $10 \mathrm{k}$ & $20 \mathrm{k}$ & $1 \mathrm{k}$ & $5 k$ & $10 \mathrm{k}$ & $20 \mathrm{k}$ \\
\hline \multirow[t]{3}{*}{512} & Max & 544 & 3142 & 5699 & 12,030 & 426 & 2319 & 3980 & 9381 \\
\hline & Min & 185 & 948 & 1587 & 2924 & 77 & 91 & 98 & 350 \\
\hline & Sum & 130,841 & 596,352 & 964,795 & $1,872,710$ & 122,359 & 523,232 & 882,270 & $1,678,095$ \\
\hline \multirow[t]{3}{*}{$1 \mathrm{k}$} & Max & 544 & 3142 & 5699 & 12,030 & 375 & 2319 & 4497 & 9021 \\
\hline & Min & 185 & 948 & 1576 & 2924 & 28 & 91 & 98 & 280 \\
\hline & Sum & 255,201 & $1,207,024$ & $1,957,944$ & $3,576,870$ & 239,219 & $1,048,327$ & $1,738,695$ & $3,224,380$ \\
\hline \multirow[t]{3}{*}{$2 \mathrm{k}$} & Max & 544 & 3142 & 5699 & 12,030 & 426 & 2319 & 4497 & 9465 \\
\hline & Min & 185 & 937 & 1576 & 2893 & 14 & 14 & 14 & 70 \\
\hline & Sum & 517,771 & $2,246,115$ & $4,057,069$ & $10,246,641$ & 477,216 & $2,026,514$ & $3,523,529$ & $5,340,693$ \\
\hline \multirow[t]{3}{*}{$4 k$} & Max & 544 & 3142 & 5699 & 12,030 & 438 & 2319 & 4553 & 9393 \\
\hline & Min & 185 & 937 & 1577 & 2893 & 14 & 14 & 14 & 70 \\
\hline & Sum & $1,047,587$ & $4,689,638$ & $7,985,342$ & $13,772,088$ & 964,999 & $4,134,084$ & $7,036,544$ & $12,523,765$ \\
\hline \multirow[t]{3}{*}{$8 \mathrm{k}$} & Max & 544 & 3142 & 5699 & 12,030 & 432 & 2364 & 4553 & 9489 \\
\hline & Min & 185 & 937 & 1577 & 2893 & 14 & 14 & 14 & 49 \\
\hline & Sum & $2,063,609$ & $9,196,415$ & $14,508,307$ & $28,847,845$ & $1,935,299$ & $8,192,319$ & $13,869,101$ & $25,545,303$ \\
\hline \multirow[t]{3}{*}{$16 \mathrm{k}$} & Max & 544 & 3142 & 5699 & 12,030 & 438 & 2364 & 4553 & 9423 \\
\hline & Min & 185 & 937 & 1576 & 2895 & 14 & 14 & 14 & 49 \\
\hline & Sum & $4,078,028$ & $17,468,936$ & $30,468,442$ & $55,962,934$ & $3,839,218$ & $16,175,042$ & $17,852,162$ & $50,669,497$ \\
\hline \multirow[t]{3}{*}{$32 \mathrm{k}$} & Max & 544 & 3142 & 5699 & 12,030 & 438 & 2364 & 4560 & 9495 \\
\hline & Min & 185 & 937 & 1576 & 2924 & 14 & 14 & 14 & 42 \\
\hline & Sum & $8,098,792$ & $38,481,691$ & $63,569,548$ & $125,007,952$ & $7,680,932$ & $32,501,442$ & $56,179,615$ & $106,443,702$ \\
\hline \multirow[t]{3}{*}{$64 \mathrm{k}$} & Max & 544 & 3142 & 5699 & 12,030 & 438 & 2364 & 4560 & 9495 \\
\hline & Min & 185 & 937 & 1576 & 2893 & 14 & 14 & 14 & 21 \\
\hline & Sum & $15,646,914$ & $77,127,273$ & $134,153,228$ & $231,739,723$ & $15,517,797$ & $67,965,278$ & $113,712,252$ & $205,476,450$ \\
\hline \multirow[t]{3}{*}{$128 \mathrm{k}$} & Max & 544 & 3142 & 5699 & 12,030 & 438 & 2364 & 4560 & 9495 \\
\hline & Min & 185 & 937 & 1576 & 2893 & 14 & 14 & 14 & 21 \\
\hline & Sum & $33,141,660$ & $148,833,606$ & $262,013,018$ & $433,884,540$ & $30,668,615$ & $132,008,892$ & $222,438,007$ & $402,884,488$ \\
\hline \multirow[t]{3}{*}{$256 k$} & Max & 544 & $3,142,937$ & 5699 & 12,030 & 438 & 2364 & 4560 & 9495 \\
\hline & Min & 185 & 937 & 1576 & 2893 & 14 & 14 & 14 & 14 \\
\hline & Sum & $71,756,336$ & $301,774,440$ & $501,780,227$ & $922,711,260$ & $57,690,021$ & $260,490,897$ & $444,624,839$ & $820,648,686$ \\
\hline
\end{tabular}


Table 5 The number of memory accesses with the FW rule set

\begin{tabular}{|c|c|c|c|c|c|c|c|c|c|}
\hline \multirow[t]{2}{*}{ Packet } & \multirow[t]{2}{*}{ Access memory } & \multicolumn{4}{|c|}{ Kd-tree algorithm } & \multicolumn{4}{|c|}{ Pruned Kd-tree algorithm } \\
\hline & & $1 \mathrm{k}$ & $5 k$ & $10 \mathrm{k}$ & $20 \mathrm{k}$ & $1 \mathrm{k}$ & $5 k$ & $10 \mathrm{k}$ & $20 k$ \\
\hline \multirow[t]{3}{*}{512} & Max & 1199 & 3130 & 5159 & 12,454 & 1033 & 2581 & 4965 & 9991 \\
\hline & Min & 422 & 1039 & 1931 & 4124 & 65 & 65 & 70 & 305 \\
\hline & Sum & 356,443 & $1,136,854$ & $1,874,853$ & $3,557,947$ & 255,322 & 853,432 & $1,457,881$ & $3,221,409$ \\
\hline \multirow[t]{3}{*}{$1 \mathrm{k}$} & Max & 1199 & 3130 & 6186 & 12,454 & 1033 & 2581 & 6163 & 9991 \\
\hline & Min & 422 & 1039 & 1931 & 4215 & 20 & 65 & 70 & 150 \\
\hline & Sum & 668,357 & $2,023,881$ & $3,210,080$ & $7,090,479$ & 549,917 & $1,840,454$ & $3,052,962$ & $6,285,888$ \\
\hline \multirow[t]{3}{*}{$2 k$} & Max & 1199 & 3130 & 6186 & 12,503 & 1086 & 2581 & 6181 & 12,496 \\
\hline & Min & 422 & 1039 & 1910 & 4124 & 10 & 65 & 70 & 50 \\
\hline & Sum & $1,341,260$ & $3,982,774$ & $6,783,093$ & $14,179,408$ & $1,072,978$ & $3,686,741$ & $6,188,172$ & $12,377,521$ \\
\hline \multirow[t]{3}{*}{$4 k$} & Max & 1199 & 3130 & 6186 & 12,503 & 1086 & 3057 & 6179 & 12,503 \\
\hline & Min & 422 & 1039 & 1910 & 4124 & 10 & 10 & 10 & 50 \\
\hline & Sum & $2,678,963$ & $7,991,672$ & $14,517,075$ & $28,159,817$ & $2,154,155$ & $7,326,447$ & $12,763,918$ & $24,901,899$ \\
\hline \multirow[t]{3}{*}{$8 \mathrm{k}$} & Max & 1199 & 3130 & 6186 & 12,503 & 1084 & 3064 & 6176 & 12,503 \\
\hline & Min & 422 & 1006 & 1910 & 4124 & 10 & 10 & 10 & 35 \\
\hline & Sum & $5,460,157$ & $16,415,274$ & $31,978,990$ & $55,019,262$ & $4,301,367$ & $14,517,171$ & $19,193,383$ & $50,439,626$ \\
\hline \multirow[t]{3}{*}{$16 k$} & Max & 1199 & 3130 & 6186 & 12,503 & 1086 & 3055 & 6180 & 12,503 \\
\hline & Min & 422 & 1006 & 1910 & 4124 & 10 & 10 & 10 & 35 \\
\hline & Sum & $10,894,541$ & $37,703,706$ & $50,566,605$ & $110,212,121$ & $8,396,090$ & $32,417,792$ & $49,128,771$ & $99,489,160$ \\
\hline \multirow[t]{3}{*}{$32 \mathrm{k}$} & Max & 1199 & 3130 & 6186 & 12,503 & 1086 & 3064 & 6180 & 12,502 \\
\hline & Min & 422 & 1006 & 1910 & 4124 & 10 & 10 & 10 & 30 \\
\hline & Sum & $23,157,719$ & $65,556,229$ & $100,929,684$ & $223,898,085$ & $16,296,924$ & $57,797,109$ & $98,517,382$ & $200,488,788$ \\
\hline \multirow[t]{3}{*}{$64 k$} & Max & 1199 & 3130 & 6186 & 12,503 & 1086 & 3064 & 6186 & 12,503 \\
\hline & Min & 422 & 1006 & 1910 & 4124 & 10 & 10 & 10 & 26 \\
\hline & Sum & $42,492,153$ & $131,326,833$ & $223,530,631$ & $455,442,469$ & $34,756,397$ & $115,950,625$ & $192,205,381$ & $398,400,842$ \\
\hline \multirow[t]{3}{*}{$128 \mathrm{k}$} & Max & 1199 & 3130 & 6186 & 12,503 & 1086 & 3064 & 6186 & 12,503 \\
\hline & Min & 422 & 1006 & 1910 & 4124 & 10 & 10 & 10 & 15 \\
\hline & Sum & $85,833,341$ & $262,031,623$ & $446,157,629$ & $891,605,570$ & $71,234,413$ & $232,741,927$ & $376,538,129$ & $788,539,632$ \\
\hline \multirow[t]{3}{*}{$256 k$} & $\operatorname{Max}$ & 1199 & 3130 & 6186 & 12,503 & 1086 & 3064 & 6186 & 12,503 \\
\hline & Min & 422 & 1006 & 1910 & 4124 & 10 & 10 & 10 & 10 \\
\hline & Sum & $175,974,550$ & $504,174,306$ & $874,611,717$ & $1,787,756,828$ & $140,608,710$ & $484,286,547$ & $775,062,307$ & $1,603,978,979$ \\
\hline
\end{tabular}

\section{Implementation and evaluation}

To implement the proposed packet classification algorithm, C++ language was used on a system with the specifications in Table 3.

The rule set and packets needed to evaluate and test the proposed algorithm was created using ClassBench tool [19]. Three general rule sets were created by ClassBench, including Access Control List (ACL), Firewall (FW), and IP Chain (IPC). We used rule sets of varying sizes for our evaluations. Each generated rule set was named according to its type and size. For example, ACL2_3K refers to an $A C L$ rule set with 3000 rules.

For our evaluations, we used rule sets of $1 \mathrm{k}$ to $20 \mathrm{k}$ and packets of 512 to $256 \mathrm{k}$. In the following, the results of testing the proposed algorithm and its comparison with the typical Kd-tree algorithm are presented and discussed.

\subsection{Number of memory accesses}

One of the criteria for evaluating packet classification algorithms is the number of memory accesses. When a packet is being classified by our method, after reading the packet header fields, the nodes of the tree are traversed. Traversal starts from the root of the tree and proceeds to the leaf node according to the search mechanism of the proposed method. If a node has rules, those rules are also checked linearly. Of course, this part of the search can be done using a hash table which reduces access time in 
Table 6 The number of memory accesses with the IPC rule set

\begin{tabular}{|c|c|c|c|c|c|c|c|c|c|}
\hline \multirow{3}{*}{$\begin{array}{l}\text { Packet } \\
512\end{array}$} & \multirow{3}{*}{$\begin{array}{l}\text { Access } \\
\text { Memory } \\
\text { Max }\end{array}$} & \multicolumn{4}{|c|}{ Kd-tree algorithm } & \multicolumn{4}{|c|}{ Pruned Kd-tree algorithm } \\
\hline & & $1 \mathrm{k}$ & $5 \mathrm{k}$ & $10 \mathrm{k}$ & $20 \mathrm{k}$ & $1 \mathrm{k}$ & $5 \mathrm{k}$ & $10 \mathrm{k}$ & $20 \mathrm{k}$ \\
\hline & & 1177 & 4505 & 8902 & 18,300 & 1035 & 4197 & 8387 & 16,768 \\
\hline & Min & 518 & 2272 & 4571 & 9258 & 24 & 52 & 56 & 244 \\
\hline & Sum & 345,996 & $1,386,816$ & $2,760,527$ & $5,594,128$ & 255,068 & $1,166,789$ & $2,373,728$ & $4,426,525$ \\
\hline \multirow[t]{3}{*}{$1 \mathrm{k}$} & Max & 1177 & 4505 & 8902 & 18,300 & 1035 & 4201 & 8393 & 16,736 \\
\hline & Min & 518 & 2272 & 4571 & 9258 & 16 & 52 & 56 & 120 \\
\hline & Sum & 672,580 & $2,933,798$ & $5,664,583$ & $11,160,473$ & 496,039 & $2,251,819$ & $4,478,732$ & $9,294,388$ \\
\hline \multirow[t]{3}{*}{$2 k$} & Max & 1177 & 4505 & 8902 & 18,300 & 1035 & 4197 & 8397 & 16,768 \\
\hline & Min & 518 & 2272 & 4571 & 9258 & 8 & 8 & 8 & 40 \\
\hline & Sum & $1,499,426$ & $5,612,115$ & $10,982,065$ & $22,795,948$ & $1,131,262$ & $4,640,361$ & $9,054,679$ & $19,039,423$ \\
\hline \multirow[t]{3}{*}{$4 k$} & Max & 1177 & 4505 & 8902 & 18,300 & 1035 & 4206 & 8394 & 16,768 \\
\hline & Min & 518 & 2272 & 4571 & 9258 & 8 & 8 & 8 & 40 \\
\hline & Sum & $2,831,981$ & $10,907,906$ & $21,219,865$ & $46,511,349$ & $1,903,639$ & $9,228,581$ & $18,675,042$ & $36,361,780$ \\
\hline \multirow[t]{3}{*}{$8 k$} & Max & 1177 & 4505 & 8902 & 18,300 & 1035 & 4209 & 8399 & 16,768 \\
\hline & Min & 518 & 2272 & 4571 & 9258 & 8 & 8 & 8 & 28 \\
\hline & Sum & $5,518,273$ & $23,191,884$ & $43,251,250$ & $89,222,546$ & $4,368,599$ & $18,740,797$ & $36,891,300$ & $73,558,586$ \\
\hline \multirow[t]{3}{*}{$16 \mathrm{k}$} & Max & 1177 & 4505 & 8902 & 18,300 & 1035 & 4209 & 8399 & 16,754 \\
\hline & Min & 518 & 2272 & 4571 & 9258 & 8 & 8 & 8 & 24 \\
\hline & Sum & $11,186,350$ & $46,906,430$ & $90,059,127$ & $179,097,795$ & $7,937,502$ & $63,000,357$ & $72,764,365$ & $148,563,167$ \\
\hline \multirow[t]{3}{*}{$32 \mathrm{k}$} & Max & 1177 & 4505 & 8902 & 18,300 & 1035 & 4209 & 8399 & 16,769 \\
\hline & Min & 518 & 2272 & 4571 & 9258 & 8 & 8 & 8 & 24 \\
\hline & Sum & $23,033,832$ & $89,320,318$ & $179,833,731$ & $370,009,708$ & $16,239,111$ & $65,876,786$ & $145,825,021$ & $291,522,203$ \\
\hline \multirow[t]{3}{*}{$64 k$} & Max & 1177 & 4505 & 8902 & 18,300 & 1035 & 4210 & 8399 & 16,769 \\
\hline & Min & 518 & 2272 & 4571 & 9258 & 8 & 8 & 8 & 12 \\
\hline & Sum & $46,402,561$ & $179,393,966$ & $358,063,296$ & $719,578,148$ & $32,402,640$ & $146,457,305$ & $291,276,079$ & $585,812,370$ \\
\hline \multirow[t]{3}{*}{$128 \mathrm{k}$} & Max & 1177 & 4505 & 8902 & 18,300 & 1035 & 4210 & 8399 & 16,769 \\
\hline & Min & 518 & 2272 & 4571 & 9258 & 8 & 8 & 8 & 12 \\
\hline & Sum & $85,383,655$ & $359,159,114$ & $715,062,556$ & $1,554,271,563$ & $61,234,320$ & $291,887,858$ & $577,484,603$ & $1,302,536,756$ \\
\hline \multirow[t]{3}{*}{$256 k$} & Max & 1177 & 4505 & 8902 & 18,300 & 1035 & 4210 & 8399 & 16,769 \\
\hline & Min & 518 & 2272 & 4571 & 9258 & 8 & 8 & 8 & 8 \\
\hline & Sum & $177,781,262$ & $762,191,145$ & $1,444,735,988$ & $2,984,245,224$ & $130,884,008$ & $579,482,047$ & $1,164,547,320$ & $2,275,663,443$ \\
\hline
\end{tabular}

examining the rules of a tuple. In this research, we have used the linear method in searching the rules of each tuple to illustrate the effect of the pruned Kd-tree. The number of memory accesses can be defined as the total number of traversed nodes along with the number of examined rules. In the following, we will examine the number of memory accesses in both Kd-tree classification algorithm and the proposed method with regard to the different rule sets and different numbers of rules and packets. Table 4 represents the number of memory accesses related to the $\mathrm{ACL}$ rule set. The table shows the lowest and highest amount of memory access per packet, and the total number of memory accesses for all the packets.

In Table 4, it can be seen that the total number of memory accesses has increased in both algorithms with the increase in the number of packets. As the number of rules increases, the number of memory accesses will increase too. In the worst case of classification, the number of accesses equals the depth of the tree plus the total number of rules available in the tuple nodes on the path from the root to the leaf. For example, with 512 packets and $1 \mathrm{k}$ rules, the Kd-tree algorithm has a maximum number of 544 memory accesses. In our method, however, the number of memory accesses decreased to 426.

On the other hand, the minimum number of memory accesses in the proposed method is less than that of the $\mathrm{Kd}$-tree algorithm. For example, in classifying $2 \mathrm{k}$ packets according to $5 \mathrm{k}$ rules, the proposed method has at least 923 less accesses to system memory than the Kd-tree algorithm. In this case, the Kd-tree algorithm makes 937 accesses, and the proposed method makes 14 accesses. 
Table 7 Classification time with the ACL rule set

\begin{tabular}{|c|c|c|c|c|}
\hline \multirow[t]{2}{*}{ Packets } & \multicolumn{4}{|l|}{ Rules } \\
\hline & $1 \mathrm{k}$ & $5 k$ & $10 \mathrm{k}$ & $20 \mathrm{k}$ \\
\hline \multicolumn{5}{|l|}{ Kd-tree } \\
\hline 512 & 52.9 & 200.1 & 426.2 & 713 \\
\hline $1 \mathrm{k}$ & 101.4 & 405.1 & 889.5 & 1442.4 \\
\hline $2 \mathrm{k}$ & 201.2 & 760.8 & 1823.2 & 3596.2 \\
\hline $4 \mathrm{k}$ & 396.5 & 1534.5 & 3614.5 & 5242 \\
\hline $8 \mathrm{k}$ & 803.3 & 3127.1 & 6540 & $10,324.8$ \\
\hline $16 \mathrm{k}$ & 1580.5 & 5726.7 & $13,749.6$ & $20,172.1$ \\
\hline $32 \mathrm{k}$ & 3157.2 & $12,477.5$ & $28,455.6$ & $44,922.5$ \\
\hline $64 k$ & 6436.6 & $25,275.4$ & $59,320.5$ & $84,939.4$ \\
\hline $128 \mathrm{k}$ & $11,682.5$ & $48,804.5$ & 115,642 & 165,533 \\
\hline $256 \mathrm{k}$ & $24,690.8$ & 135,354 & 218,268 & 352,803 \\
\hline \multicolumn{5}{|c|}{ Pruned Kd-tree } \\
\hline 512 & 46 & 174.9 & 396.3 & 644.1 \\
\hline $1 \mathrm{k}$ & 93.4 & 350.9 & 784.1 & 1113.5 \\
\hline $2 \mathrm{k}$ & 177.5 & 669.2 & 1579.9 & 2078 \\
\hline $4 k$ & 373.1 & 1399.5 & 3168 & 4609.1 \\
\hline $8 k$ & 720 & 2698.2 & 6222.8 & 9177.8 \\
\hline $16 \mathrm{k}$ & 1532.7 & 5332.9 & $12,485.9$ & $18,873.7$ \\
\hline $32 \mathrm{k}$ & 2957.4 & $10,803.6$ & $25,162.8$ & $39,105.3$ \\
\hline $64 k$ & 5804.4 & $22,255.2$ & $50,568.6$ & $73,975.3$ \\
\hline $128 \mathrm{k}$ & $11,023.2$ & 47,713 & $99,622.1$ & 158,810 \\
\hline $256 \mathrm{k}$ & $19,848.4$ & 118,386 & 196,271 & 318,850 \\
\hline
\end{tabular}

Table 5 shows the number of memory accesses for classifying the packets corresponding to the $\mathrm{FW}$ rule set in the two algorithms. In our evaluations, the number of required accesses to system memory for packet classification was examined. Similar to the results in Table 4, for this rule set the pruned $\mathrm{Kd}$-tree method makes fewer accesses to the system memory than the Kd-tree algorithm. It should be noted that memory access has a direct relationship with the time of classification. In other words, increased memory access would increase the time of classification and decreased access would decrease it.

Similarly, Table 6 lists the number of memory accesses in packet classification with IPC rule set. According to the results, with an increased number of packets and rules, the number of memory accesses has also increased. The reason why an increased number of rules augmented memory access is the enlargement of the tree of rules. As the rules increase in number, the number of tuples and, accordingly, the number of rules in each tuple will also increase. According to Table 6, for example, for $16 \mathrm{k}$ packets and $1 \mathrm{k}$ rules in the $\mathrm{Kd}$-tree algorithm, the number of memory accesses is 10894541 , but it reaches 50566605 with increasing the number of rules to $10 \mathrm{k}$.
Table 8 Classification time with the FW rule set

\begin{tabular}{|c|c|c|c|c|}
\hline \multirow[t]{2}{*}{ Packets } & \multicolumn{4}{|l|}{ Rules } \\
\hline & $1 \mathrm{k}$ & $5 \mathrm{k}$ & $10 \mathrm{k}$ & $20 \mathrm{k}$ \\
\hline \multicolumn{5}{|l|}{ Kd-tree } \\
\hline 512 & 132.5 & 420.9 & 703.8 & 1540.9 \\
\hline $1 \mathrm{k}$ & 248.6 & 780.2 & 1206.8 & 3068.1 \\
\hline $2 \mathrm{k}$ & 515.3 & 1716.8 & 2532.9 & 5897.3 \\
\hline $4 k$ & 1003.1 & 3369.3 & 5423.6 & $11,591.8$ \\
\hline $8 \mathrm{k}$ & 2188.8 & 6424.8 & $12,028.9$ & $20,572.5$ \\
\hline $16 \mathrm{k}$ & 4201.4 & $14,302.9$ & $19,089.8$ & $37,940.3$ \\
\hline $32 \mathrm{k}$ & 8975 & $25,120.4$ & $39,453.4$ & $71,972.3$ \\
\hline $64 \mathrm{k}$ & $16,773.1$ & $47,973.5$ & $86,182.2$ & 144,052 \\
\hline $128 \mathrm{k}$ & $33,901.9$ & $95,255.8$ & 178,078 & 355,075 \\
\hline $256 \mathrm{k}$ & $70,610.1$ & 190,010 & 322,950 & 792,204 \\
\hline \multicolumn{5}{|c|}{ Pruned Kd-tree } \\
\hline 512 & 95.3 & 325.9 & 549.9 & 1472 \\
\hline $1 \mathrm{k}$ & 203.9 & 703.5 & 1148.7 & 2603.7 \\
\hline $2 \mathrm{k}$ & 408.7 & 1526.8 & 2344.4 & 5067.9 \\
\hline $4 \mathrm{k}$ & 865.2 & 2874.6 & 4776.4 & $10,498.2$ \\
\hline $8 \mathrm{k}$ & 1694 & 5603.6 & 9161.1 & $17,849.7$ \\
\hline $16 \mathrm{k}$ & 3248.1 & $12,302.7$ & $18,752.2$ & $35,544.4$ \\
\hline $32 k$ & 6385.6 & $21,266.7$ & $38,853.2$ & $65,392.3$ \\
\hline $64 \mathrm{k}$ & $14,620.1$ & $42,197.7$ & $74,233.1$ & 137,763 \\
\hline $128 \mathrm{k}$ & 27,829 & $86,457.6$ & 144,751 & 317,864 \\
\hline $256 \mathrm{k}$ & $54,544.8$ & 181,531 & 274,718 & 720,966 \\
\hline
\end{tabular}

From the results in Tables 4, 5 and 6 it can be concluded that, in the classification of different numbers of input packets with rules of varying type and size, memory access in the pruned Kd-tree algorithm is less than in the typical $\mathrm{Kd}$-tree algorithm. These results are indicative of the better performance of the proposed method. For example, given an IPC rule set of $20 \mathrm{k}$ rules and $256 \mathrm{k}$ packets, the total number of memory accesses for the Kd-tree algorithm is 2984245224 . In this case, the number of memory accesses by the proposed method is 2275663443 . Therefore, our method reduces memory access by $23.74 \%$. The main reason for this reduction is the fact that right-hand leaves are pruned and the rules in every tuple are sorted.

\subsection{Packet classification time}

The time interval from the moment the packets arrive at the classifier until they are classified by the algorithm is called packet classification time. In this section, the packet classification time for different rule sets is examined. Packet classification time in this experiment was measured in milliseconds

Table 7 shows the classification time with the $A C L$ rule set. According to this table, with an increase in the 
Table 9 Classification time with the IPC rule set

\begin{tabular}{|c|c|c|c|c|}
\hline \multirow[t]{2}{*}{ Packets } & \multicolumn{4}{|l|}{ Rules } \\
\hline & $1 \mathrm{k}$ & $5 \mathrm{k}$ & $10 \mathrm{k}$ & $20 k$ \\
\hline \multicolumn{5}{|l|}{ Kd-tree } \\
\hline 512 & 148.8 & 459.9 & 1236 & 2122 \\
\hline $1 \mathrm{k}$ & 284.4 & 972.7 & 2540.5 & 4719.8 \\
\hline $2 \mathrm{k}$ & 589.5 & 1798.5 & 4890.7 & 9237.7 \\
\hline $4 k$ & 1091.9 & 3507.2 & 9352.5 & $18,086.1$ \\
\hline $8 \mathrm{k}$ & 2134.1 & 8296.3 & $19,648.5$ & $35,600.5$ \\
\hline $16 \mathrm{k}$ & 4409.8 & $14,584.2$ & $33,420.1$ & $72,090.5$ \\
\hline $32 \mathrm{k}$ & 9116.4 & $28,521.4$ & $64,648.1$ & 143,146 \\
\hline $64 k$ & 17,619 & $61,756.7$ & 148,144 & 260,777 \\
\hline $128 \mathrm{k}$ & 34,130 & 123,424 & 274,478 & 532,779 \\
\hline $256 \mathrm{k}$ & $66,588.8$ & 341,769 & 548,962 & $1,080,750$ \\
\hline \multicolumn{5}{|c|}{ Pruned Kd-tree } \\
\hline 512 & 112.5 & 379.2 & 1073.6 & 1787.3 \\
\hline $1 \mathrm{k}$ & 197.4 & 718 & 2015.5 & 3812.2 \\
\hline $2 \mathrm{k}$ & 449.9 & 1506.5 & 4101.3 & 7638.7 \\
\hline $4 k$ & 744.7 & 3000.8 & 8376.3 & $14,331.5$ \\
\hline $8 \mathrm{k}$ & 1643.4 & 6022 & $16,213.2$ & $29,138.5$ \\
\hline $16 \mathrm{k}$ & 3110.6 & $11,856.4$ & $26,599.6$ & 57,428 \\
\hline $32 \mathrm{k}$ & 6324.8 & $21,253.3$ & $59,890.8$ & 113,045 \\
\hline $64 k$ & $12,109.5$ & $52,257.6$ & 118,218 & 223,633 \\
\hline $128 \mathrm{k}$ & $23,178.5$ & 112,349 & 211,450 & 443,647 \\
\hline $256 \mathrm{k}$ & $51,236.3$ & 261,962 & 464,276 & 992,319 \\
\hline
\end{tabular}

number of packets, packet classification time in both the basic and pruned algorithms would increase. With any number of packets, the proposed method has a shorter classification time than the Kd-tree algorithm. For example, the classification time for $32 \mathrm{k}$ packets is $3157.2 \mathrm{~ms}$ in the $\mathrm{Kd}$-tree algorithm and $2957.4 \mathrm{~ms}$ in the proposed method. Therefore, our method reduces the time by $199.8 \mathrm{~ms}$.

Also, with all the different rule sets, the proposed algorithm has consumed less time than the Kd-tree algorithm. With $10 \mathrm{k}$ rules, for example, the speed of the proposed algorithm for classifying $64 \mathrm{k}$ packets is $14.75 \%$ higher than the Kd-tree algorithm. With $20 \mathrm{k}$ rules, the proposed algorithm has classified $2 \mathrm{k}$ packets in $2078 \mathrm{~ms}$ whereas the typical Kd-tree algorithm has classified this number of packets in $2596.2 \mathrm{~ms}$. In other words, the Kd-tree algorithm is $24.94 \%$ slower than the proposed algorithm.

In the next experiment, packet classification time with FW and IPC rule sets was measured. Tables 8 and 9 represent the results for $1 \mathrm{k}, 5 \mathrm{k}, 10 \mathrm{k}$, and $20 \mathrm{k}$ rules. In this rule set, too, the packet classification time of the proposed method in all cases is much less than that of the Kd-tree algorithm. For example, with an IPC rule set of $1 \mathrm{k}$ rules, $256 \mathrm{k}$ packets are classified in $66,588.8 \mathrm{~ms}$ by the $\mathrm{Kd}$-tree algorithm and in $51,236.3 \mathrm{~ms}$ by our proposed algorithm. Therefore, the proposed method functions $15,352.5 \mathrm{~ms}$ faster. This difference is also seen in other results.

Overall, the proposed method provided us with a shorter classification time with all rule sets (i.e., $\mathrm{ACL}, \mathrm{FW}$, and IPC) in comparison with the typical Kd-tree algorithm. The results obtained in this section are consistent with the results of examining the number of memory accesses, which is indicative of the superiority of the proposed method.

\subsection{Throughput}

Throughput refers to the number of packets that are classified in the unit of time. Increased throughput means that more packets have been classified in a second.

Figure 9a-d depict the throughput of algorithms on the $A C L$ rule set with $1 k, 5 k, 10 k$, and $20 k$ rules, respectively. As the created tree enlarges with an increase in the number of rules, the packet classification time will also increase. As a result, the number of packets classified in a second will be reduced. Our results show that throughput with smaller rule sets is higher than throughput with larger rule sets. For example, the throughput of the Kd-tree algorithm in the classification of $32 \mathrm{k}$ packets is 10,135 packets per second with $1 \mathrm{k}$ rules and 1124 packets per second with $10 \mathrm{k}$ rules. With $\mathrm{ACL}$ rule set, our method had a higher throughput than the Kd-tree algorithm in all classification scenarios. In Fig. 9a, the throughput of classifying $8 \mathrm{k}$ packets is 9958.92 packets per second for the Kd-tree algorithm and $11,111.11$ packets per second for the proposed method.

Figure 10a-d show the results of the throughput of the algorithms for classification of packets based on FW rule set with $1 k, 5 k, 10 k$, and $20 k$ rules, respectively. In this rule set, too, the proposed method has a higher throughput than the Kd-tree algorithm. With all the numbers of packets and rules, the proposed method classifies more packets than the Kd-tree algorithm in one second. For example, Fig. 10a shows that the Kd-tree algorithm has resulted in a throughput of 3864.15 packets per second in classifying $512 \mathrm{k}$ packets with $1 \mathrm{k}$ rules. However, our proposed method has achieved a rate of 5372.51 packets per second in the same scenario. In other words, our method classifies $39.03 \%$ more packets in the same time interval.

The maximum throughput obtained by our method with FW rule set was 5372.51 packets per second with $1 \mathrm{k}$ rules, 1571.03 packets per second with $5 \mathrm{k}$ rules, 931.86 packets per second with $10 \mathrm{k}$ rules, and 489.35 packets per second with $20 \mathrm{k}$ rules. As discussed above, decreased throughput is due to the increased complexity of the Kdtree or its corresponding pruned tree which increases traversal time. The throughput obtained by the $\mathrm{Kd}$-tree algorithm in classifying $4 \mathrm{k}$ packets was 3987.64 packets 


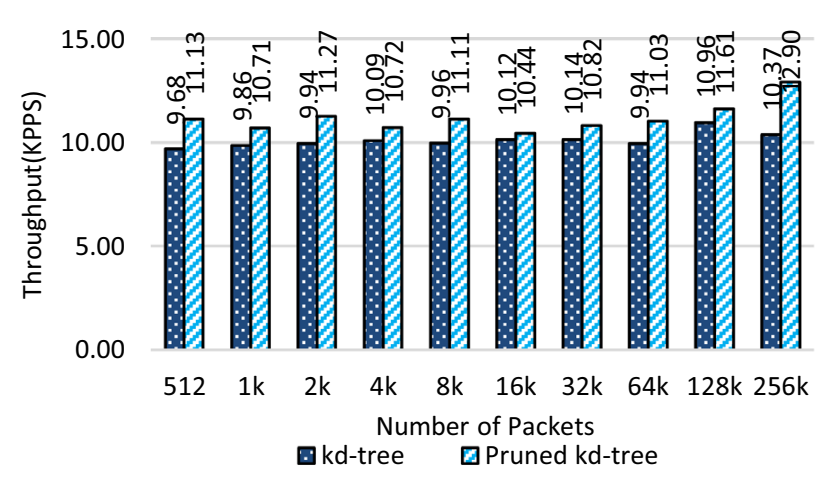

(a) ACL - $1 \mathrm{k}$ rules

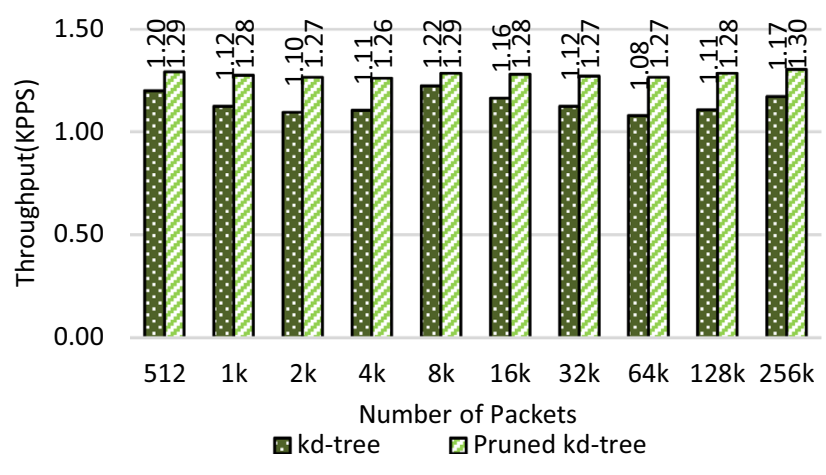

(c) ACL - 10k rules

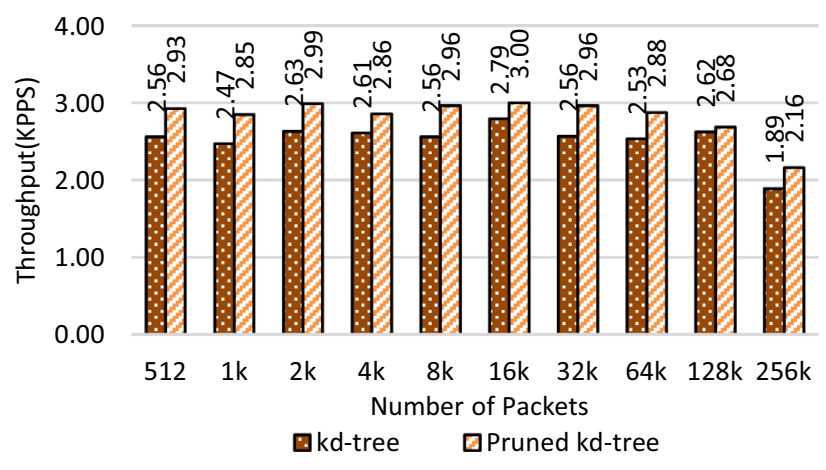

(b) ACL - $5 \mathrm{k}$ rules

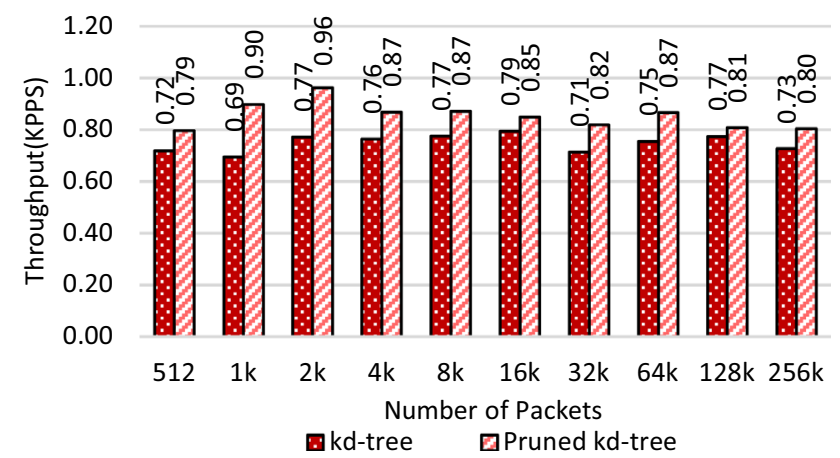

(d) ACL - 20k rules

Fig. 9 Throughput of the kd-tree algorithm and the proposed method with different rule sets in ACL rules

per second with $1 \mathrm{k}$ rules, 1187.19 packets per second with $5 \mathrm{k}$ rules, 737.52 packets per second with $10 \mathrm{k}$ rules, and 345.07 packets per second with $20 \mathrm{k}$ rules.

The proposed method and the Kd-tree algorithm were implemented on IPC rule set. The results of this experiment on $1 \mathrm{k}, 5 \mathrm{k}, 10 \mathrm{k}$, and $20 \mathrm{k}$ rules are shown in Fig. $11 \mathrm{a}-\mathrm{d}$, respectively. With this rule set, too, the proposed method obtained a higher throughput rate than the Kd-tree algorithm. According to Fig. 11a, with 64k packets, our method classified 5285.11 packets per second while the Kd-tree algorithm classified 3632.44 packets per second. In other words, our method classified 1652.67 more packets per second. Here again, the number of rules increased due to the enlarged and more complicated tree, thereby reducing the throughput rate.

Overall, the maximum throughput value obtained by the proposed method with ACL, FW, and IPC rule sets was $12897.77,5372.51$, and 5522.36 packets per second. However, the maximum throughput obtained by the basic algorithm with the same rule sets was 10,956.56, 4022.53, and 3844.49, respectively. Moreover, in all scenarios, the number of packets classified by the proposed method was much more than those classified by the Kdtree algorithm at the same time.

\subsection{Memory usage for the tree structure}

One of the important criteria in comparing the efficiency of the proposed method and the basic algorithm is the amount of memory needed to store the tree structure created by the rules. The data structure used to hold the tree is composed of the following parts:

- Nodes that hold the tuples.

- Nodes that hold the number of member tuples and the number of markers assigned to the tuple.

Figure 12 shows the memory consumption in bytes of the Kd-tree algorithm and the proposed method with $A C L$, FW, and IPC rule sets. In the graphs of Fig. 12, the memory needed to hold tuple nodes and rules separately, together with the sum of the two, is shown for both algorithms. Graphs (a) through (d) show the 


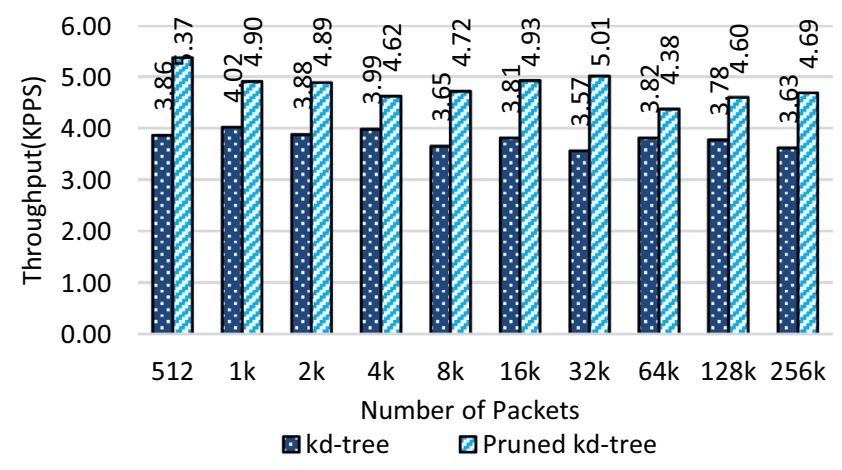

(a) $\mathrm{FW}-1 \mathrm{k}$ rules

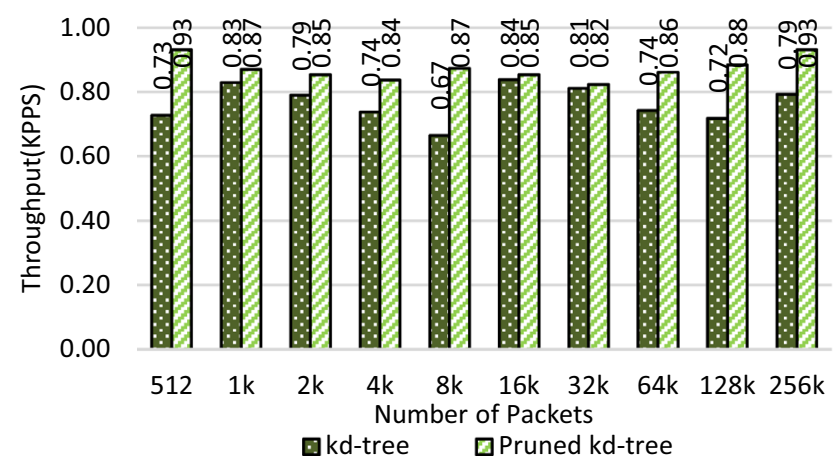

(c) $\mathrm{FW}-10 \mathrm{k}$ rules

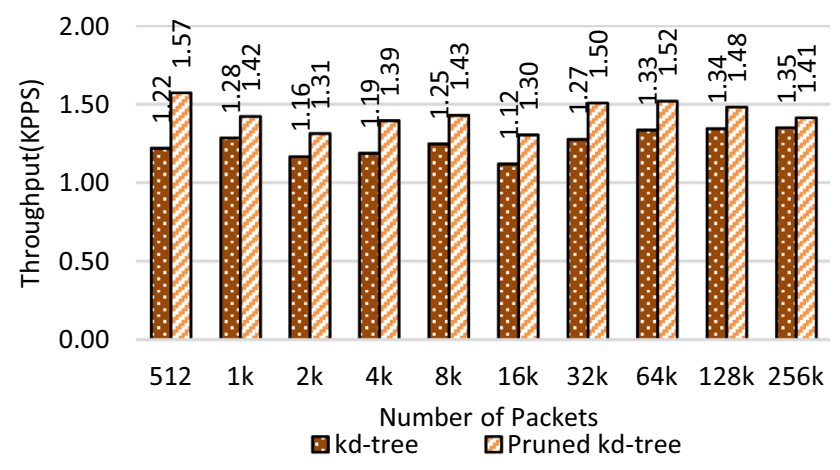

(b) FW - 5k rules

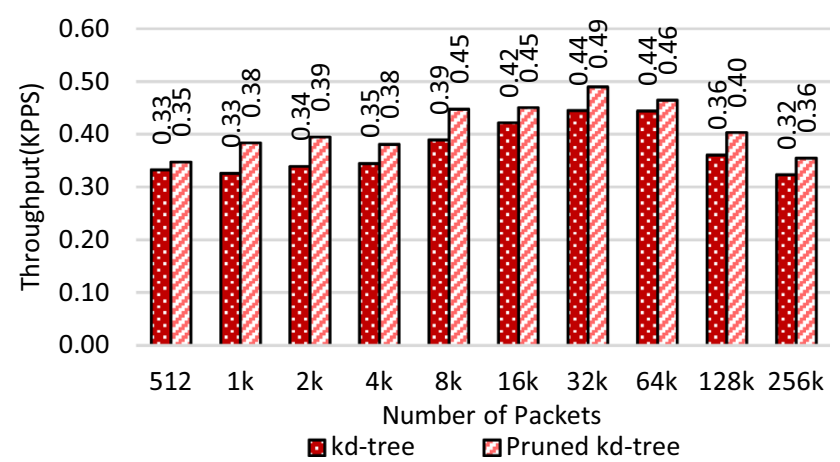

(d) $\mathrm{FW}-1 \mathrm{k}$ rules

Fig. 10 Throughput of the kd-tree algorithm and the proposed method with different rule sets in FW rules

amount of memory needed to store the data structure corresponding to $1 \mathrm{k}, 5 \mathrm{k}, 10 \mathrm{k}$, and $20 \mathrm{k}$ rules, respectively.

According to the results of this test, the proposed method requires less memory in all scenarios. For example, with an ACL rule set of $1 \mathrm{k}$ rules, the memory needed to store the $\mathrm{Kd}$-tree is $23,815.9$ bytes while it is $19,120.4$ bytes in the proposed method. Therefore, the proposed method was able to reduce memory usage by 4695.5 bytes through pruning the tree nodes. With an FW rule set of $10 \mathrm{k}$ rules, the $\mathrm{Kd}$-tree algorithm needs $0.74 \mathrm{~KB}$ for storing nodes that contain tuples and $156.09 \mathrm{~KB}$ for storing nodes that contain rules. By pruning the redundant nodes, however, the proposed method needs only $0.54 \mathrm{~KB}$ for tuple nodes and 127.21 KB for nodes that contain rules. With IPC rule set, too, our method used less memory than the Kd-tree algorithm. This confirms the success of the method in pruning the $\mathrm{Kd}$-tree data structure and making better use of memory.

Overall, our method consumed less memory than the $\mathrm{Kd}$-tree algorithm with all rule sets and all numbers of rules. In other words, our method for pruning the Kd-tree was efficient and could have a positive effect on reducing the size of the tree.

Table 10 shows the comparison of the proposed method with other packet classification algorithms. The algorithms in the first column are compared according to three evaluation criteria including lookup time, memory usage, and dimension scalability.

First, the lookup time is examined. Most of the existing designs have a time complexity of either $O(W) \operatorname{or} O(\log N)$. Four algorithms provide better search efficiency. The time complexity of RFC [20] and HiCuts [21] are constant. The binary search scheme [22] has a time complexity of $O\left(\log ^{2} W\right)$. RFC and HiCuts have lower time complexity but at the high cost of considerable memory requirement, i.e. $O\left(N^{d}\right)$. Excluding RFC and HiCuts, our design has the lowest time complexity $O\left(d \log \left(\frac{w}{\alpha}\right)\right)$ with controlled storage space requirement $O\left(N \log \left(\frac{w}{\alpha}\right)\right)$. Here, $1 \ll \alpha \ll W$ represents the minimum ratio of the reduction in any of the distinct traversal patches from the root to any leaf node of the tree. 


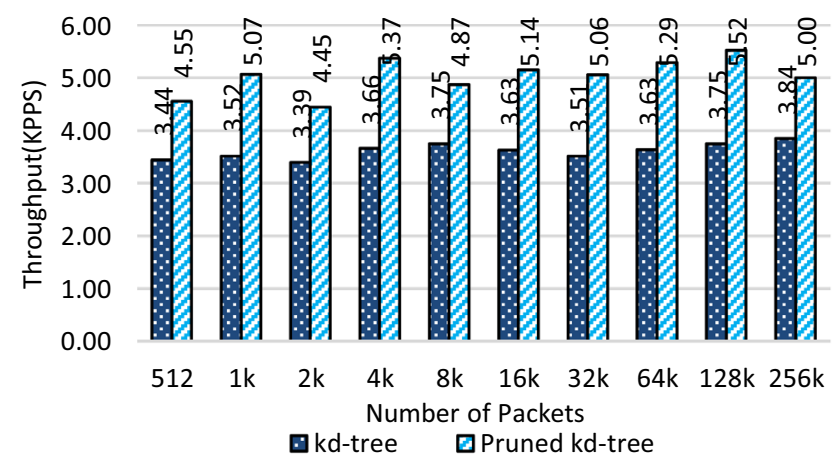

(a) IPC - $1 \mathrm{k}$ rules

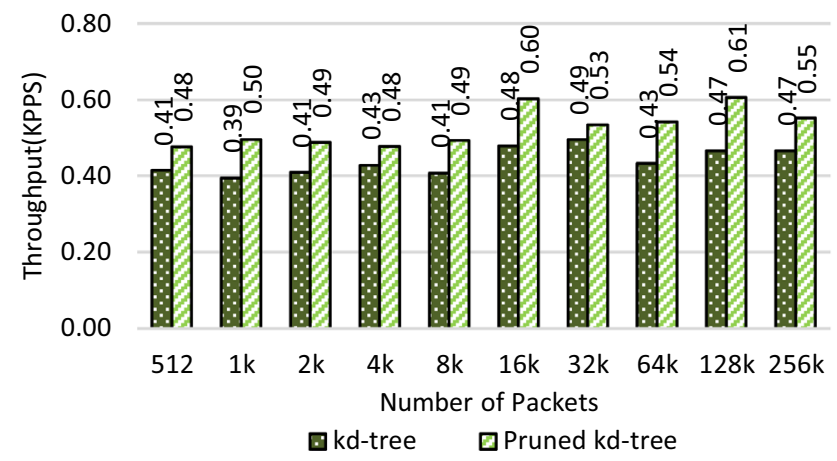

(c) IPC - 10k rules

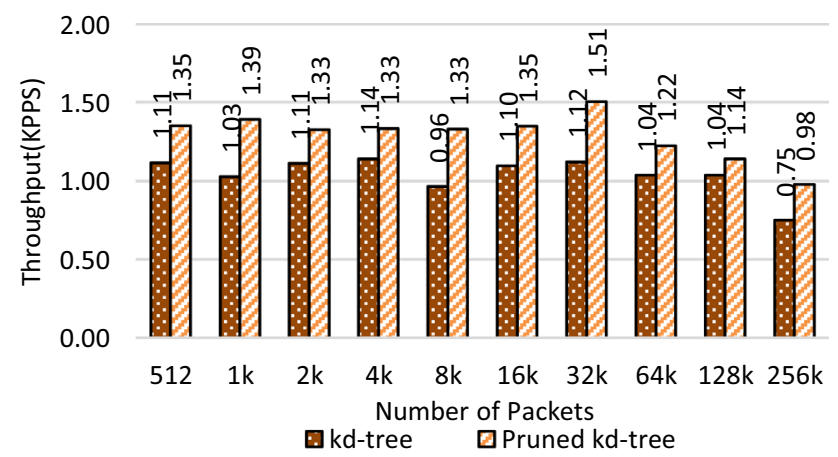

(b) IPC - $5 \mathrm{k}$ rules

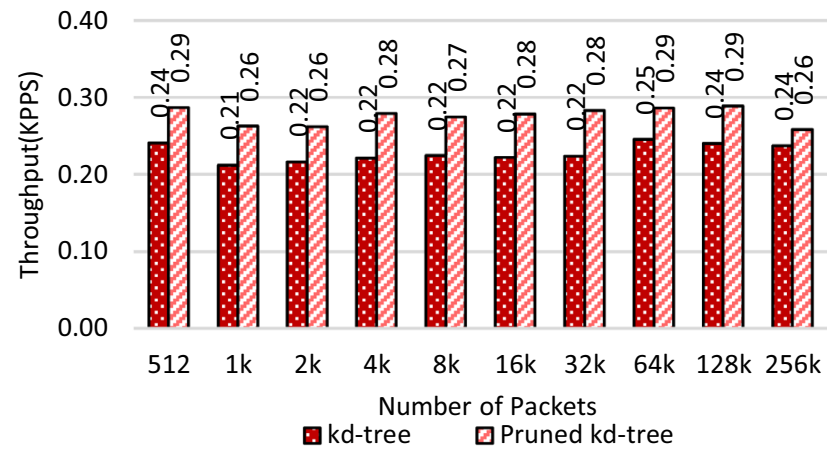

(d) IPC - 20k rules

Fig. 11 Throughput of the kd-tree algorithm and the proposed method with different rule sets in IPC rules

Second, we compare the proposed design with Rectangle Search and Binary Search, which are both based on tuple space search. The two schemes can only be applied to two-dimensional classifiers, that is $d=2$. Rectangle Search requires $O(W)$ hashes and Binary Search needs $O\left(\left(\log _{2} W\right)^{2}\right)$ hashes. The proposed design only requires $O(2 \log W)$ hashes. From the storage perspective, Rectangle Search requires a storage space of $O(N W)$ and Binary Search requires a space of $O\left(N * \log ^{2} W\right)$. In contrast, our design only needs $O\left(N \log \left(\frac{w}{\alpha}\right)\right)$ of memory.

\section{Conclusion}

Packet classification plays a crucial role in the efficiency of many Internet-based processing devices such as routers and firewalls. Different software and hardware algorithms perform packet classification. One of the well-known algorithms for packet classification is the Kd-tree algorithm. By dividing the rules based on the length of the prefix of source and destination IP addresses, this algorithm places them in tuples. Then it sorts tuples with a special technique and creates a binary tree using the sorted tuples.
The input packets of the classifier can be classified using a search algorithm on this tree. The Kd-tree algorithm has several disadvantages including:

- Lack of order in the rules of the tuples and the necessity to examine all the rules of a tuple during classification.

- Existence of redundant nodes in the tree.

- Repetitive checking of rules during packet classification.

Given the defects in the Kd-tree algorithm, we attempted to develop an optimized version of the algorithm by close analysis of its structure as well as how it traverses the tree structure. The proposed method improves the $\mathrm{Kd}$-tree algorithm in terms of both time and memory consumption. The key idea behind the optimized version is to prune redundant nodes that do not affect the process of classification. This can reduce memory access as well as the memory required for storing the tree structure. Also, in the proposed algorithm, sorting the rules in a tuple according to their priority has led to a further reduction in the classification time.

Both the proposed method and the Kd-tree algorithm were implemented on three rule sets, i.e., $\mathrm{ACL}$, FW, and 


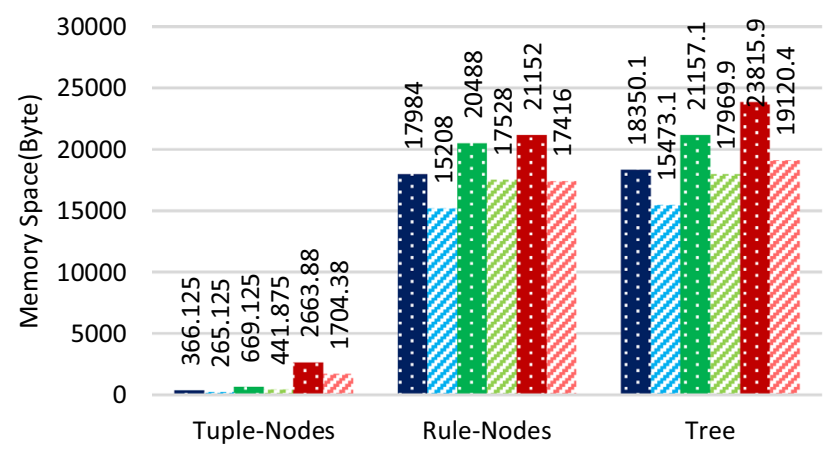

(a) $1 \mathrm{k}$ Rules

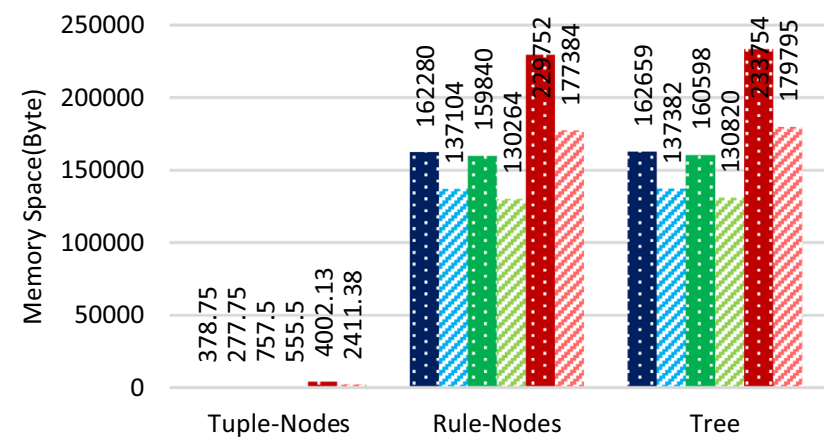

(c) $10 \mathrm{k}$ Rules

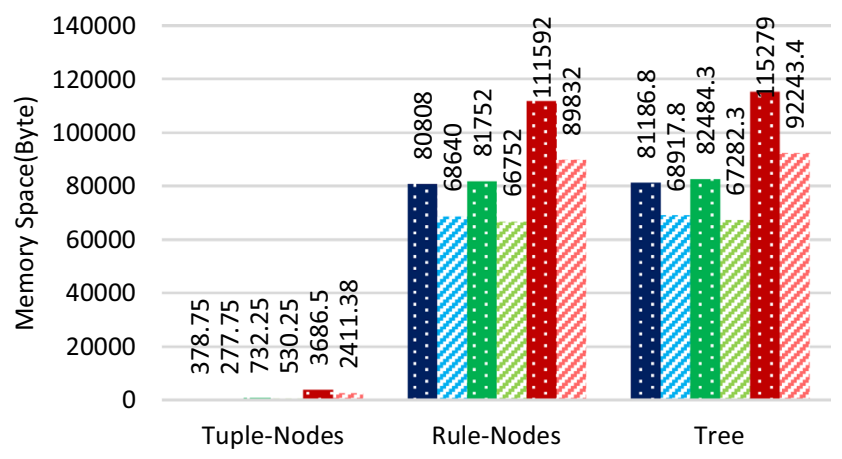

(b) $5 \mathrm{k}$ Rules

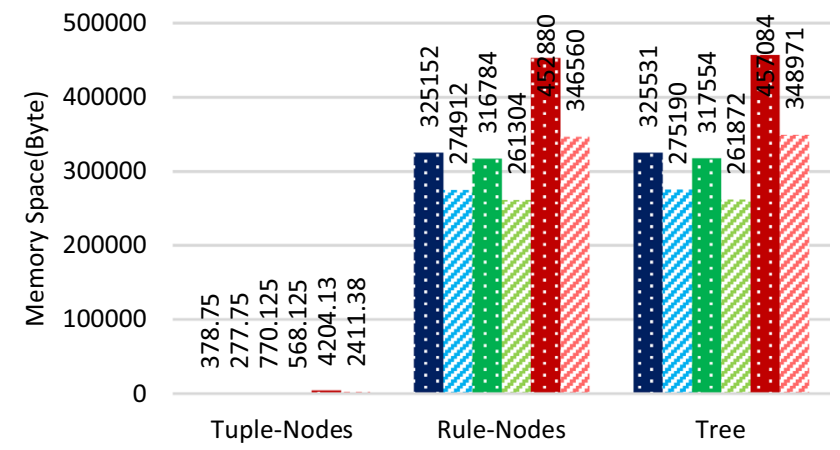

(d) $20 \mathrm{k}$ Rules

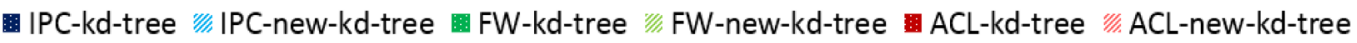

Fig. 12 Memory required for tree structure

Table 10 The complexity of different algorithms

\begin{tabular}{lll}
\hline Evaluation algorithms & Lookup time & Memory usage \\
\hline Linear search on filter rules & $O(N)$ & $O(N)$ \\
Grid-of-tries [23] & $O(W)$ & $O(N W)$ \\
Cross-producting [24] & $O(d W)$ & $O\left(N^{d}\right)$ \\
Bit-parallelism [25] & $O(W \log N)$ & $O(N W)$ \\
Area-based QuadTree [26] & $O(W)$ & $O(N W)$ \\
Fat-Inverted segment tree [27] & $O((L+1) W)$ & $O\left(L N^{(1+1 / L)}\right)$ \\
Segment tree with fractional cascading [28] & $O(\log N)$ & $O(N * \log N)$ \\
Recursive flow classification [20] & $O(d)$ & $O\left(N^{d}\right)$ \\
HiCuts [21] & $O(d)$ & $O\left(N^{d}\right)$ \\
Linear search on tuple [29] & $O\left(W^{d}\right)$ & $O(N)$ \\
Rectangle search [30] & $O(W)$ & $O(N W)$ \\
Binary search [22] & $\left.O(\log )^{2} W\right)$ & $O\left(N \log { }^{2} W\right)$ \\
Extended Grid-of-Trie [31] & $O(W)$ & $O(N W)$ \\
Kd-tree [6] & $O(d \log W)$ & $O(N d \log W)$ \\
Proposed method & $O\left(d \log \left(\frac{W}{\alpha}\right)\right)$ & $O\left(N \log \left(\frac{w}{\alpha}\right)\right)$ \\
\end{tabular}


IPC. The rule sets were created using Classbench tool. This tool is used to generate experimental rules and packets for testing classification systems. The evaluation criteria we used include the number of tuples created by the rules, the number of memory accesses, packet classification time, throughput, memory consumption of the tree data structure, and the depth of the tree.

According to the results, the proposed method has been more efficient than the Kd-tree algorithm in every aspect. According to the results of the classification of $128 \mathrm{k}$ headers with IPC $1 \mathrm{k}$ rule set, our proposed algorithm could boost the classification speed of the basic $\mathrm{Kd}$-tree algorithm by a ratio 1.47 . Furthermore, our method needs less memory space than the Kd-tree algorithm for storing the tree data structure. The depth of the pruned tree is less than that of the Kd-tree algorithm.

One suggestion to continue the present research with the aim of reducing the classification time is to develop a method for searching the rules in a tuple. Currently, the rules of a tuple are checked linearly. In the worst case, this may require the input packet to be matched against all the rules. The time complexity of this search operation is in the worst case $O(n)$, where $n$ denotes the number of rules in the tuple. Therefore, if the time for checking the rules can be reduced, the classification time will also be reduced. For this purpose, like other methods derived from the basic algorithm, we can use hashing methods. Thus, the number of memory accesses to find the best matching rule in each tuple would decrease significantly.

\section{Compliance with ethical standards}

Conflict of interest The authors declare that they have no conflict of interest.

\section{References}

1. Hager S, John P, Dietzel S, Scheuermann B (2018) RuleBender: Tree-based policy transformations for practical packet classification systems. Comput Netw 135:253-265

2. Inoue T, Mano T, Mizutani K, Minato S-I, Akashi O (2018) Fast packet classification algorithm for network-wide forwarding behaviors. Comput Commun 116:101-117

3. Cheng Y-C, Wang P-C (2015) Packet classification using dynamically generated decision trees. IEEE Trans Comput 64:582-586

4. Sun P, Lan J, Wang P, Ma T (2017) RFC: range feature code for TCAM-based packet classification. Comput Netw 118:54-61

5. Qi Y, Xu L, Yang B, Xue Y, Li J (2009) Packet classification algorithms: from theory to practice. IEEE INFOCOM 2009:648-656

6. Shieh S, Lee F-Y, Lin Y-W (2004) Accelerating network security services with fast packet classification. Comput Commun 27:1637-1646

7. Gupta P, McKeown N (1999) Packet classification using hierarchical intelligent cuttings. In: Hot interconnects VII
8. Singh S, Baboescu F, Varghese G, Wang J (2003) Packet classification using multidimensional cutting. In: Proceedings of the 2003 conference on applications, technologies, architectures, and protocols for computer communications, pp 213-224

9. Qi Y, Fong J, Jiang W, Xu B, Li J, Prasanna V (2010) Multi-dimensional packet classification on FPGA: $100 \mathrm{Gbps}$ and beyond. In: International conference on field-programmable technology, pp 241-248

10. Bentley JL (1975) Multidimensional binary search trees used for associative searching. Commun ACM 18:509-517

11. Robinson JT (1981) The KDB-tree: a search structure for large multidimensional dynamic indexes. In: Proceedings of the 1981 ACM SIGMOD international conference on management of data, pp 10-18

12. Guttman A (1984) R-trees: a dynamic index structure for spatial searching, vol 14. ACM, New York

13. Sellis T, Roussopoulos N, Faloutsos C (1987) The R+-tree: a dynamic index for multi-dimensional objects

14. Beckmann N, Kriegel H-P, Schneider R, Seeger B (1990) The $R^{*}$-tree: an efficient and robust access method for points and rectangles. In: ACM sigmod record, pp 322-331

15. Ahn HK, Mamoulis N, Wong HM (2001) A survey on multidimensional access methods. Technical report, Institute of Infomation and ComputingSciences, Utrecht University, The Netherlands

16. Böhm C, Berchtold S, Keim DA (2001) Searching in highdimensional spaces: index structures for improving the performance of multimedia databases. ACM Comput Surv (CSUR) 33:322-373

17. Brown L, Gruenwald L (1998) Tree-based indexes for image data. J Vis Commun Image Represent 9:300-313

18. Gaede V, Günther O (1998) Multidimensional access methods. ACM Comput Surv (CSUR) 30:170-231

19. Taylor DE, Turner JS (2007) Classbench: a packet classification benchmark. IEEE/ACM Trans Netw 15:499-511

20. Li X, Shao Y (2018) Memory compression for recursive flow classification algorithm in network packet processing devices. In: IEEE 3rd advanced information technology, electronic and automation control conference (IAEAC), pp 1502-1505

21. Chang Y-K, Chen H-C (2018) Fast packet classification using recursive endpoint-cutting and bucket compression on FPGA. Comput J 62:198-214

22. Baboescu F, Warkhede P, Suri S, Varghese G (2006) Fast packet classification for two-dimensional conflict-free filters. Comput Netw 50:1831-1842

23. Nottingham A, Irwin B (2009) GPU packet classification using OpenCL: a consideration of viable classification methods. In: Proceedings of the 2009 annual research conference of the South African Institute of Computer Scientists and Information Technologists, pp 160-169

24. Pao D, Lu Z (2014) A multi-pipeline architecture for high-speed packet classification. Comput Commun 54:84-96

25. Lakshman T, Stiliadis D (1998) High-speed policy-based packet forwarding using efficient multi-dimensional range matching. In: ACM SIGCOMM computer communication review, pp 203-214

26. Lee J, Byun H, Mun JH, Lim H (2017) Utilizing 2-D leaf-pushing for packet classification. Comput Commun 103:116-129

27. Feldman A, Muthukrishnan S (2000) Tradeoffs for packet classification. In: Proceedings IEEE INFOCOM 2000. Conference on computer communications. Nineteenth annual joint conference of the IEEE computer and communications societies (Cat. No. 00CH37064), pp 1193-1202

28. Su C-F (2000) High-speed packet classification using segment tree. In: Globecom'00-IEEE. Global telecommunications conference. Conference record (Cat. No. 00CH37137), pp 582-586 
29. Daly J, Bruschi V, Linguaglossa L, Pontarelli S, Rossi D, Tollet J et al (2019) TupleMerge: fast software packet processing for online packet classification. In: IEEE/ACM transactions on networking

30. Srinivasan V, Suri S, Varghese G (1999) Packet classification using tuple space search. In: ACM SIGCOMM computer communication review, pp 135-146
31. Erdem O (2016) Pipelined hierarchical architecture for high performance packet classification. Comput Netw 103:143-164

Publisher's Note Springer Nature remains neutral with regard to jurisdictional claims in published maps and institutional affiliations. 